\title{
GASP XXIII: A Jellyfish Galaxy as an Astrophysical Laboratory of the Baryonic Cycle
}

\author{
Bianca M. Poggianti ${ }^{1}$ (D), Alessandro Ignesti ${ }^{2,14}$, Myriam Gitti $^{2,14}$ (D), Anna Wolter ${ }^{3}$, Fabrizio Brighenti ${ }^{2,14}$, Andrea Biviano ${ }^{4}$ (D), \\ Koshy George $^{5,15,16}$ (D), Benedetta Vulcani ${ }^{1}$ (D), Marco Gullieuszik ${ }^{1}$ (D), Alessia Moretti ${ }^{1}$ (D), Rosita Paladino ${ }^{6}$, Daniela Bettoni ${ }^{1}$ (D), \\ Andrea Franchetto $^{1,7}$, Yara L. Jaffé ${ }^{8}$ (D), Mario Radovich ${ }^{1}$, Elke Roediger ${ }^{9}$, Neven Tomičić ${ }^{1}$ (D), Stephanie Tonnesen ${ }^{10}$ (D), \\ Callum Bellhouse ${ }^{11}$ (D), Jacopo Fritz ${ }^{12}$ (D), and Alessandro Omizzolo ${ }^{1,13}$ \\ ${ }^{1}$ INAF-Padova Astronomical Observatory, Vicolo dell'Osservatorio 5, I-35122 Padova, Italy; bianca.poggianti@inaf.it \\ ${ }^{2}$ Dipartimento di Fisica e Astronomia, Universita' di Bologna, via Gobetti 93/2, I-40129 Bologna, Italy \\ ${ }^{3}$ INAF-Brera Astronomical Observatory, via Brera 28, I-20121 Milano, Italy \\ ${ }^{4}$ INAF, Astronomical Observatory of Trieste, via Tiepolo 11, I-34131 Trieste, Italy \\ ${ }^{5}$ Faculty of Physics, Ludwig-Maximilians-Universität, Scheinerstr. 1, D-81679, Munich, Germany \\ ${ }^{6}$ INAF-Istituto di Radioastronomia, via P. Gobetti 101, I-40129 Bologna, Italy \\ ${ }^{7}$ Dipartimento di Fisica e Astronomia, vicolo dell'Osservatorio 5, I-35136 Padova, Italy \\ ${ }^{8}$ Instituto de Fisica y Astronomia, Universidad de Valparaiso, Avda. Gran Bretana 1111 Valparaiso, Chile \\ ${ }^{9}$ E.A. Milne Centre for Astrophysics, Department of Physics and Mathematics, University of Hull, Hull, HU6 7RX, UK \\ ${ }^{10}$ Flatiron Institute, CCA, 162 5th Avenue, New York, NY 10010, USA \\ ${ }^{11}$ University of Birmingham School of Physics and Astronomy, Edgbaston, Birmingham, UK \\ ${ }^{12}$ Instituto de Radioastronomia y Astrofisica, UNAM, Campus Morelia, AP 3-72, CP 58089, Mexico \\ ${ }^{13}$ Vatican Observatory, Vatican City, Vatican State \\ ${ }^{14}$ INAF, Istituto di Radioastronomia di Bologna, via Gobetti 101, I-40129 Bologna, Italy \\ 15 Department of Physics, Christ University, Hosur Road, Bangalore 560029, India \\ ${ }^{16}$ Indian Institute of Astrophysics, Koramangala II Block, Bangalore, India \\ Received 2019 July 26; revised 2019 October 22; accepted 2019 October 25; published 2019 December 17
}

\begin{abstract}
With MUSE, Chandra, VLA, ALMA, and UVIT data from the GASP program, we study the multiphase baryonic components in a jellyfish galaxy (JW100) with a stellar mass $3.2 \times 10^{11} M_{\odot}$ hosting an active galactic nucleus (AGN). We present its spectacular extraplanar tails of ionized and molecular gas, UV stellar light, and $\mathrm{X}$-ray and radio continuum emission. This galaxy represents an excellent laboratory to study the interplay between different gas phases and star formation and the influence of gas stripping, gas heating, and AGNs. We analyze the physical origin of the emission at different wavelengths in the tail, in particular in situ star formation (related to $\mathrm{H} \alpha, \mathrm{CO}$, and $\mathrm{UV}$ emission), synchrotron emission from relativistic electrons (producing the radio continuum), and heating of the stripped interstellar medium (ISM; responsible for the X-ray emission). We show the similarities and differences of the spatial distributions of ionized gas, molecular gas, and UV light and argue that the mismatch on small scales $(1 \mathrm{kpc})$ is due to different stages of the star formation process. We present the relation $\mathrm{H} \alpha$-X-ray surface brightness, which is steeper for star-forming regions than for diffuse ionized gas regions with a high $[\mathrm{O} \mathrm{I}] / \mathrm{H} \alpha$ ratio. We propose that ISM heating due to interaction with the intracluster medium (either for mixing, thermal conduction, or shocks) is responsible for the X-ray tail, observed [O I] excess, and lack of star formation in the northern part of the tail. We also report the tentative discovery in the tail of the most distant (and among the brightest) currently known ULX, a pointlike ultraluminous X-ray source commonly originating in a binary stellar system powered by either an intermediatemass black hole or a magnetized neutron star.
\end{abstract}

Unified Astronomy Thesaurus concepts: Galaxy evolution (594); Galaxy clusters (584); Galaxy processes (614)

\section{Introduction}

Ram pressure stripping is considered the most efficient mechanism to remove gas from galaxies in galaxy clusters (Boselli \& Gavazzi 2006). A multitude of observational studies have observed the smoking gun of this physical process at various wavelengths with different techniques, mostly $\mathrm{H} \mathrm{I}, \mathrm{H} \alpha$ narrowband imaging, UV/blue light, and, more recently, integral field spectroscopy (Kenney et al. 2004; Chung et al. 2007; Hester et al. 2010; Smith et al. 2010; Yagi et al. 2010; Merluzzi et al. 2013; Kenney et al. 2014; Fossati et al. 2016; Consolandi et al. 2017; Gullieuszik et al. 2017; Jáchym et al. 2017; Moretti et al. 2018; Bellhouse et al. 2019; Fossati et al. 2019).

The most extreme examples of galaxies undergoing strong ram pressure are the so-called "jellyfish galaxies" (Smith et al. 2010; Ebeling et al. 2014; Fumagalli et al. 2014). They have extraplanar, unilateral debris visible in the optical/UV light and striking tails of $\mathrm{H} \alpha$ ionized gas. Most of the $\mathrm{H} \alpha$ emission in the tails is due to photoionization by massive stars born in situ in the tail in dynamically quite cold $\mathrm{H} \alpha$-emitting clumps resembling giant and supergiant $\mathrm{H}$ II regions and complexes (Poggianti et al. 2019, and references therein), with possibly some exceptions (e.g., NGC 4569; Boselli et al. 2016).

Optical line ratio diagnostic diagram maps obtained with integral field spectroscopy show that ionization mechanisms other than in situ star formation (SF) are also at play in the tails, contributing especially to the interclump diffuse emission (Fossati et al. 2016; Poggianti et al. 2019). Different optical line ratios depict a generally consistent picture but provide significantly different values for the fraction of tail emission due to SF or shocks/heating.

In Poggianti et al. (2019), we studied the optical ionization mechanisms in the tails of a significant sample of jellyfish galaxies (16 in total) from the GAs Stripping Phenomena in 
galaxies survey (GASP $;{ }^{17}$ Poggianti et al. 2017b), finding that the tail emission characteristics of the jellyfish galaxy JW100 are peculiar. At odds with the majority of the other jellyfish galaxies, SF is not the obviously dominant ionization mechanism of the tail: according to the [O III] $5007 / \mathrm{H} \beta$ versus [O I] 6300/H $\alpha$ diagnostic diagram, it has only a few starforming clumps in the tail and large amounts of ionized gas with an [O I] 6300 line excess.

A high [O I] 6300/H $\alpha$ ratio is usually interpreted as a sign of the presence of shocks (Rich et al. 2011), and shock-heated molecular hydrogen has been observed with Spitzer in some cluster galaxies undergoing ram pressure stripping (Sivanandam et al. 2010, 2014; Wong et al. 2014). Thermal heating of the stripped gas where this meets the hot intracluster medium (ICM) is another possible source of ionization, and its relevance might depend on the local ICM conditions, which can be studied with X-ray observations. The exact source of the [O I] excitation in jellyfish tails is currently unknown. Understanding why JW100 is so special in its tail ionization mechanism might be the key to understanding under what conditions stars are forming in the tails and when they are not.

The interaction with the hot X-ray-emitting ICM is expected to be crucial to set the conditions of the gas in the tails. Such interaction might give rise to an X-ray tail (Sun et al. 2010), but so far, there are only a few X-ray-emitting ram pressure stripped tails observed. There are deep Chandra archive data for JW100, as well as a set of multiwavelength data obtained by the GASP project, and it therefore offers a great opportunity to study the relation between the properties of the stripped gas tail and those of the ICM. The only other jellyfish for which a comparably rich multiwavelength data set is available is ESO 137-001, a low-mass $\left((5-8) \times 10^{9} M_{\odot}\right)$ galaxy in the A3627 cluster with $\mathrm{H} \alpha$ and other optical emission lines, molecular gas, and X-ray tails (Sun et al. 2007, 2010; Sivanandam et al. 2010; Fumagalli et al. 2014; Fossati et al. 2016; Jachym et al. 2019). Apart from JW100 and ESO 137-001, there are X-ray studies for ESO 137-002 (also in A3627; Sun et al. 2010) and UGC 6697 in A1367 (Sun \& Vikhlinin 2005) and weak/shorter tails reported in NGC 4438 and NGC 4388 in Virgo and NGC 4848 in Coma (Sun et al. 2010), plus an X-ray map of D100 in Coma shown in Jáchym et al. (2017). No strong X-ray tail has been detected in the Virgo cluster, and Sun et al. (2010) and Tonnesen et al. (2011) explained this evidence with the fact that the X-ray tail luminosity should increase with the ambient pressure, which is not very high in Virgo.

The aim of this work is to shed some light on the physical mechanisms that create tails observable at different wavelengths in jellyfish galaxies, with the ultimate goal of understanding when the process of ram pressure stripping in clusters can be observed in any given range of the electromagnetic spectrum. To do this, we perform a simultaneous analysis of the multiwavelength data set collected for JW100 by GASP. In Section 2 we introduce the galaxy JW100 and summarize its properties based on previous studies, describing its host cluster and location within the cluster in Section 2.1. Section 3 presents all of the data used in this paper: MUSE, Atacama Large Millimeter/submillimeter Array (ALMA), Very Large Telescope (VLA), Chandra (Section 3.1), and the UV Imaging Telescope (UVIT; Section 3.2). The results of a detailed analysis of the X-ray data are shown in Section 4. In

\footnotetext{
${ }^{17} \mathrm{http}: / /$ web.oapd.inaf.it/gasp/index.html
}

Section 5 we discuss the spatial distribution and physical origin of the emission at different wavelengths: optical emission lines, CO, and UV in Section 5.1; radio continuum in Section 5.2 and $\mathrm{X}$-ray in Section 5.3. The X-ray point sources, namely, the active galactic nucleus (AGN) and ultraluminous X-ray source (ULX) candidate, are discussed in Section 6. Our results are summarized in Section 7.

In this paper, we use a Chabrier (2003) initial mass function (IMF) and the standard concordance cosmology parameters $H_{0}=70 \mathrm{~km} \mathrm{~s}^{-1} \mathrm{Mpc}^{-1}, \Omega_{M}=0.3$, and $\Omega_{\Lambda}=0.7$. At the JW100 cluster redshift $(z=0.05509)$, this yields $1^{\prime \prime}=$ $1.071 \mathrm{kpc}$. The galaxy itself has a redshift $z=0.06189$.

\section{The Galaxy}

Also known as IC 5337, JW100 is an almost edge-on spiral galaxy in the cluster A2626 (Figure 1, Table 1). ${ }^{18}$ Selected by Poggianti et al. (2016) as a stripping candidate, it is one of the GASP jellyfish galaxies with the most striking ionized gas tails and the most massive galaxy of the GASP sample with a stellar mass $3.2 \times 10^{11} M_{\odot}$ (Poggianti et al. 2017b). ${ }^{19}$ The stellar and ionized gas kinematics obtained with MUSE were presented in Poggianti et al. (2017b), and a visual 3D representation can be seen at https://web.oapd.inaf.it/gasp/jw100.html.

Hosted in JW100 is a central AGN (Seyfert 2) that is detectable both in the X-ray (Wong et al. 2008) and from MUSE emission line ratios (Poggianti et al. 2017b; see also ESO press release No. 1725, https://www.eso.org/public/ news/eso1725/). A detailed MUSE analysis confirms that AGN photoionization models are required to explain its emission line properties in the central region (Radovich et al. 2019). This work has also found a biconical outflow extending for $\sim 2.5 \mathrm{kpc}$ in the northwest-to-southeast direction with a velocity offset of $\sim 250 \mathrm{~km} \mathrm{~s}^{-1}$ and a bolometric AGN luminosity estimated from the luminosity of the [O III] 5007 line of $10^{43.9} \mathrm{erg} \mathrm{s}^{-1}$ (Radovich et al. 2019). The derived mass outflow rate is low, $<0.01 M_{\odot} \mathrm{yr}^{-1}$, in agreement with what is observed in AGNs of similar luminosity. In Section 6 we will provide the AGN X-ray luminosity.

Poggianti et al. (2019) computed JW100's current SF rate (SFR) from the $\mathrm{H} \alpha$ luminosity corrected for both stellar absorption and dust extinction using the Balmer decrement adopting the Kennicutt (1998) relation $\left(\operatorname{SFR}\left(M_{\odot} \mathrm{yr}^{-1}\right)=4.6 \times\right.$ $\left.10^{-42} L_{\mathrm{H} \alpha}\left(\mathrm{erg} \mathrm{s}^{-1}\right)\right)$ and including only those spaxels that, according to the [O III] $5007 / \mathrm{H} \beta$ versus [S II] $717,6731 / \mathrm{H} \alpha$ diagram, are ionized by SF. We found a total (disk+tail) current SFR $=4.0 M_{\odot} \mathrm{yr}^{-1}$, of which $20 \%$ is in the tail. Its mass and SFR place JW100 about 0.4 dex below the SFR-mass relation for normal galaxies and $\sim 0.65$ dex below the relation for jellyfish galaxies (Vulcani et al. 2018), indicating that SF has already decreased due to gas stripping. When using the [O III] 5007/H $\beta$ versus [O I] 6300/ $\mathrm{H} \alpha$ diagnostic diagram, due to the excess of [O I] 6300 in the areas of diffuse emission in

\footnotetext{
${ }^{18}$ Some works in the literature refer to JW 100 as an S0 galaxy (e.g., Wong et al. 2008). Our analysis of the MUSE $I$-band light profile shows a type II disk (Freeman 1970; Erwin et al. 2008) and favors the hypothesis this is a (perhaps early) spiral (A. Franchetto et al. 2019, in preparation). Moreover, our stellar population analysis with the SINOPSIS code (Fritz et al. 2017) shows that SF was significant and widespread throughout the disk until the ram pressure started to strip the gas, as happens in spirals. However, given its inclination (about $75^{\circ}$ ), it is hard to assign a robust Hubble type.

${ }^{19}$ We note that the stellar mass was estimated in a slightly different manner in other papers, but the values are consistent within the errors, i.e., Vulcani et al. (2018), Poggianti et al. (2019).
} 


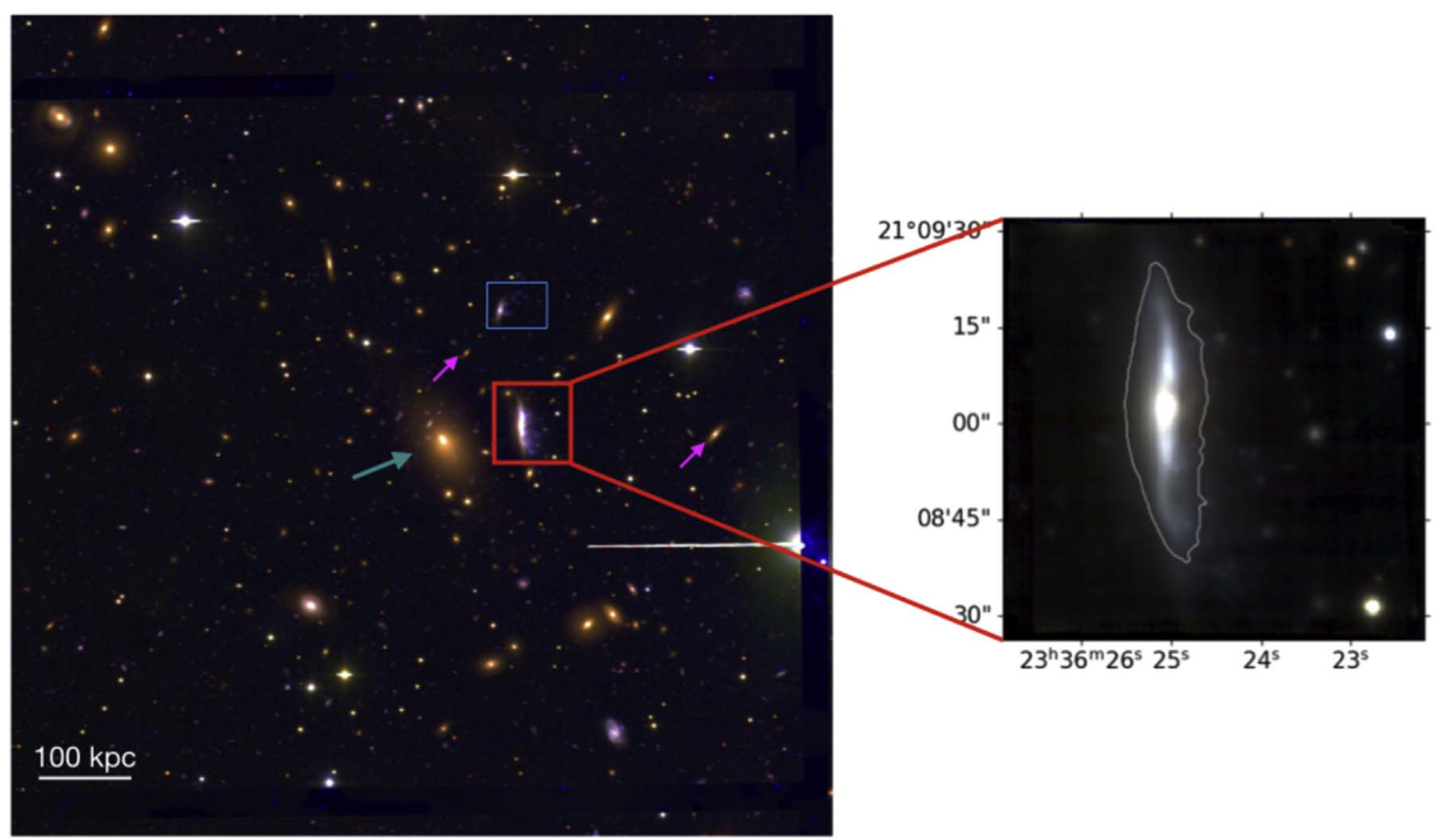

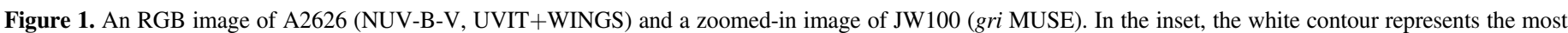

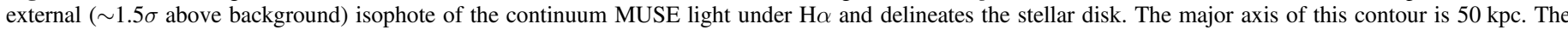

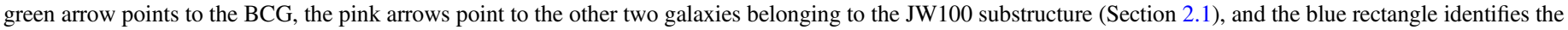
other jellyfish, JW103.

the tail (Figure 2; see Section 1), we find that the total SFR is only SFR $=2.0 M_{\odot} \mathrm{yr}^{-1}$, of which only $4 \%$ is in the tail. The different conclusions reached using different optical emission lines will be discussed throughout the paper.

\subsubsection{JW100 in Its Cluster Environment}

A rather poor cluster, A2626 has an estimated X-ray luminosity of $1.9 \times 10^{44} \mathrm{erg} \mathrm{s}^{-1}$ (Wong et al. 2008), a velocity dispersion $\sigma=650_{-49}^{+53} \mathrm{~km} \mathrm{~s}^{-1}$, and a mass $M_{200}=3.9_{-0.7}^{+1.5} \times$ $10^{14} M_{\odot}$ (Biviano et al. 2017). This cluster hosts a peculiar radio continuum emission with arc-like features that appear quite symmetric around the cluster central cD galaxy. The origin of this emission, also known as the "Kite radio source," is still unknown (Gitti et al. 2004; Gitti 2013; Ignesti et al. 2017, 2018; Kale \& Gitti 2017). Here JW100 is placed within A2626 in the most favorable conditions for ram pressure stripping (Jaffé et al. 2018), with a very high line-of-sight velocity relative to the cluster mean $\left(1807 \mathrm{~km} \mathrm{~s}^{-1}\right.$ in the cluster rest frame) and a projected distance from the cluster center (taken to coincide with the brightest cluster galaxy (BCG), the cD galaxy IC 5338) of only $83 \mathrm{kpc}$ (Figure 3 ). We note that $\sim 150 \mathrm{kpc}$ to the north of JW100, there is another jellyfish candidate, JW103 (Poggianti et al. 2016, and Figure 1), and the $\mathrm{cD}$ is also a very peculiar object, with a double nucleus and an AGN in the southern nucleus.

Using the OmegaWINGS spectroscopic catalog of galaxies in the A2626 field (Moretti et al. 2017), we identify 92 members with a new membership algorithm (CLUMPS; E. Munari et al. 2019, in preparation) based on the location of gaps in velocity space. We then run the DS+ method of Biviano et al. (2017) on the selected cluster members to detect cluster substructures. We detect six substructures that contain 21 cluster members in total. The galaxy JW100 belongs to one of these substructures, a group of three galaxies located at a median distance of $100 \pm 62 \mathrm{kpc}$ from the cluster center, with a median cluster rest-frame velocity of $1628 \pm 100 \mathrm{~km} \mathrm{~s}^{-1}$ and a velocity dispersion ${ }^{20}$ of $145_{-55}^{+79} \mathrm{~km} \mathrm{~s}^{-1}$ (see Figure 1). In Figure 3, we display the projected phase-space distribution of the cluster members within and outside substructures. The projected position and velocity of JW100 suggest that this galaxy is falling at a very high speed into A2626 for the first time on a radial orbit in the direction opposite to the observer, and it is likely close to pericentric passage (Jaffé et al. 2018). In addition, the extended tails visible in the plane of the sky indicate that the true velocity of the galaxy in the cluster must be higher than the (already high) measured line-of-sight velocity.

Given the small projected distance between JW100 and the $\mathrm{BCG}$, it is worth pondering the importance of gravitational interactions between the two galaxies. First of all, it is worth noting that neither the deep optical MUSE image (see Figure 1) nor the JW100 stellar velocity dispersion map (Figure 1 in Poggianti et al. 2017b) indicate a significant disturbance. The optical image shows a warped regular disk, and the stellar velocity map displays a regular, undisturbed rotating disk. Second, the line-of-sight velocity of JW100 relative to that of the BCG is $1772 \mathrm{~km} \mathrm{~s}^{-1}$ (from MUSE data of both); therefore, this could only be a very high speed encounter. Moreover, our SINOPSIS spectrophotometric code does not detect a significant population of extraplanar old stars, which should be present if the extraplanar material were due to tidal effects. Furthermore, a crude approach to estimate the relative importance of the tidal acceleration $a_{\text {tid }}$ from a close neighbor versus the acceleration of the attraction from the galaxy itself $a_{\text {gal }}$ can be obtained following Vollmer et al. (2005), as $a_{\mathrm{tid}} / a_{\mathrm{gal}}=M_{\mathrm{BCG}} / M_{\mathrm{JW} 100}(d / R-1)^{-2}$, where $R$ is the distance

\footnotetext{
${ }^{20}$ Being based on only three values, these statistical estimates must be considered very tentative.
} 
Table 1

JW100 Properties ${ }^{\mathrm{a}}$

\begin{tabular}{lccccccc}
\hline \hline $\begin{array}{l}\text { ID } 16 \\
(1)\end{array}$ & $\begin{array}{c}\text { Cluster } \\
(2)\end{array}$ & $\begin{array}{c}z_{\text {gal }} \\
(3)\end{array}$ & $\begin{array}{c}z_{\text {clu }} \\
(4)\end{array}$ & $\begin{array}{c}\sigma_{\text {clu }}\left(\mathrm{km} \mathrm{s}^{-1}\right) \\
(5)\end{array}$ & $\begin{array}{c}\text { R.A.(J2000) } \\
(6)\end{array}$ & $\begin{array}{c}\text { Decl.(J2000) } \\
(7)\end{array}$ & $\begin{array}{c}M_{*}\left(M_{\odot}\right) \\
(8)\end{array}$ \\
\hline JW100 & A2626 & 0.06189 & 0.05509 & $650_{-49}^{+53}$ & $23: 36: 25.054$ & $+21: 09: 02.64$ \\
\hline
\end{tabular}

${ }^{a}$ Columns are (1) GASP ID number from Poggianti et al. (2016), (2) host cluster, (3) galaxy redshift, (4) cluster redshift from Moretti et al. (2014), (5) cluster velocity dispersion from Biviano et al. (2017), (6) and (7) galaxy R.A. and decl., and (8) galaxy stellar mass.

from the center of the galaxy, and the BCG stellar mass $M_{\mathrm{BCG}}=7.8 \times 10^{11} M_{\odot}$ was estimated using literature absolute magnitude values and the Bell \& de Jong (2001) formulation. Assuming as a distance between the galaxies the projected distance $d \geqslant 83 \mathrm{kpc}$ (which is a lower limit), the tidal acceleration is smaller than the gravitational acceleration from the galaxy itself out to $R=32 \mathrm{kpc}$, larger than the stripping radius. Finally, the one-sided ionized gas tail and its direction with respect to the BCG disfavor the tidal hypothesis. Although a mild tidal interaction cannot be excluded, we conclude that ram pressure stripping plays a major role for the points addressed in this paper.

\section{Data}

In this paper we use MUSE, ALMA, VLA, Chandra, and UVIT data of JW100.

This galaxy was observed as part of the GASP program with the MUSE spectrograph in wide-field mode on 2016 July 15 with $1^{\prime \prime}$ seeing, covering a $1^{\prime} \times 1^{\prime}$ field of view with 0 ." $2 \times 0$ ". 2 pixels and a spectral range between 4800 and $9300 \AA$ sampled at $1.25 \AA$ pixel $^{-1}$ with a spectral resolution FWHM $=2.6 \AA$ (Bacon et al. 2010). The MUSE observations, data reduction, and methods of analysis are described in Poggianti et al. (2017a). In the following, we will use the $\mathrm{H} \alpha$ emission (top left panel of Figure 7) measured from the MUSE data cube corrected for both Galactic foreground and intrinsic dust extinction using the measured Balmer decrement and underlying stellar absorption using our SINOPSIS stellar population code (Fritz et al. 2017). Moreover, we will use the ionization mechanism classification based on the [O III] $5007 / \mathrm{H} \beta$ versus [S II] 6717,6731/H $\alpha$ and versus [O I] 6300/ $\mathrm{H} \alpha$ diagrams (Figure 2) taken from Poggianti et al. (2019). ${ }^{21}$

Within the GASP project, JW100 has also been observed with ALMA during Cycle 5. Observations in Bands $3(\sim 100$ $\mathrm{GHz})$ and $6(\sim 220 \mathrm{GHz})$ were taken to sample the $\mathrm{CO}(1-0)$ and $\mathrm{CO}(2-1)$ lines, respectively, achieving in both bands a resolution of $\sim 1^{\prime \prime}$ and an rms in $20 \mathrm{~km} \mathrm{~s}^{-1}$ wide channels of $\sim 0.8 \mathrm{mJy} \mathrm{beam}^{-1}$. The details of the ALMA observations, data reduction, and analysis can be found in Moretti et al. (2019). In this paper, we use the Band 6 data combined with additional Atacama Compact Array (ACA) observations, sampling the angular scale from $1^{\prime \prime}$ to $26^{\prime \prime}$.

We use the $1.4 \mathrm{GHz}$ A- and B-configuration VLA data from a project focused on the peculiar Kite radio source located at the center of A2626 (project code AG795; PI: Gitti). These observations and the data reduction are described in Gitti (2013). The VLA map at $1.4 \mathrm{GHz}$ with a resolution of 3 !" $8 \times 3$ !" 4 and an $\mathrm{rms}$ of $15.6 \mu \mathrm{Jy}$ beam $^{-1}$ is presented in the bottom right panel of Figure 7, where only regions at least $3 \sigma$ above the rms are displayed. By starting from the calibrated

\footnotetext{
21 The diagnostic diagram based on the [N II] $6583 / \mathrm{H} \alpha$ ratio is not examined due to contamination by a sky line.
}

visibilities of Gitti (2013), we produced this image with Common Astronomy Software Application (CASA) v4.7 by setting the visibility weighting to NATURAL and adopting a tapering of the baselines within $90 \mathrm{k} \lambda$ to enhance the sensitivity to the diffuse emission.

\subsection{Chandra}

In two projects focused on A2626 in January 2003 (ObsID 3192; PI: C. Sarazin, 25 ks exposure time) and 2013 October (ObsID 16136; PI: C. Sarazin, 110 ks exposure time), JW100 was observed by the Chandra X-ray Observatory. The observations were made with the ACIS-S instrument in VFAINT mode. We retrieved the data sets from the Chandra archive $^{22}$ and reprocessed them with CIAO 4.10 and CALDB 4.8.1 to correct for known time-dependent gain and charge transfer inefficiency. In order to filter out strong background flares, we also applied screening of the event files. ${ }^{23}$

For the background subtraction, we used the CALDB "Blank-Sky" files normalized to the count rate of the source image in the $10-12 \mathrm{keV}$ band. Finally, we identified the point sources using the CIAO task WAVDETECT, with the detection threshold set to the default value of $10^{-6}$ as a probability to find a spurious source. To improve absolute astrometry, we crossmatched the point sources identified in both data sets with the optical catalog USNO-A2.0 and then produced a mosaic of the two observations with the merge_obs script.

The exposure-corrected, background-subtracted Chandra mosaic in the $0.5-2.0 \mathrm{keV}$ energy band with a resolution of $\sim 0$. 5 is shown in the bottom left panel of Figure 7.

\subsection{UVIT}

The UV imaging of the GASP project is from the UVIT on board the Indian multiwavelength astronomy satellite AstroSat (Agrawal 2006). In this paper, we use near-UV (NUV) imaging observations taken with the $\mathrm{N} 242 \mathrm{~W}$ broadband filter with an angular resolution of $\sim 1$ !"2 (Tandon et al. 2017a). The NUV image is corrected for distortion (Girish et al. 2017), flatfielding, and satellite drift using the software CCDLAB (Postma \& Leahy 2017). The final image created is for a net integration of 10,106.64 $\mathrm{s}$ and is presented in the top right panel of Figure 7. The astrometric calibration is performed using the astrometry.net package, where solutions are performed using the USNO-B catalog (Lang et al. 2010). The photometric calibration is done using the zero-point values generated for photometric calibration stars as described in Tandon et al. (2017b). The UV data for JW100 and other GASP jellyfish galaxies will be used for a detailed analysis in a later paper.

\footnotetext{
22 http://cxc.harvard.edu/cda/

23 http://cxc.harvard.edu/ciao/guides/acis_data.html
} 

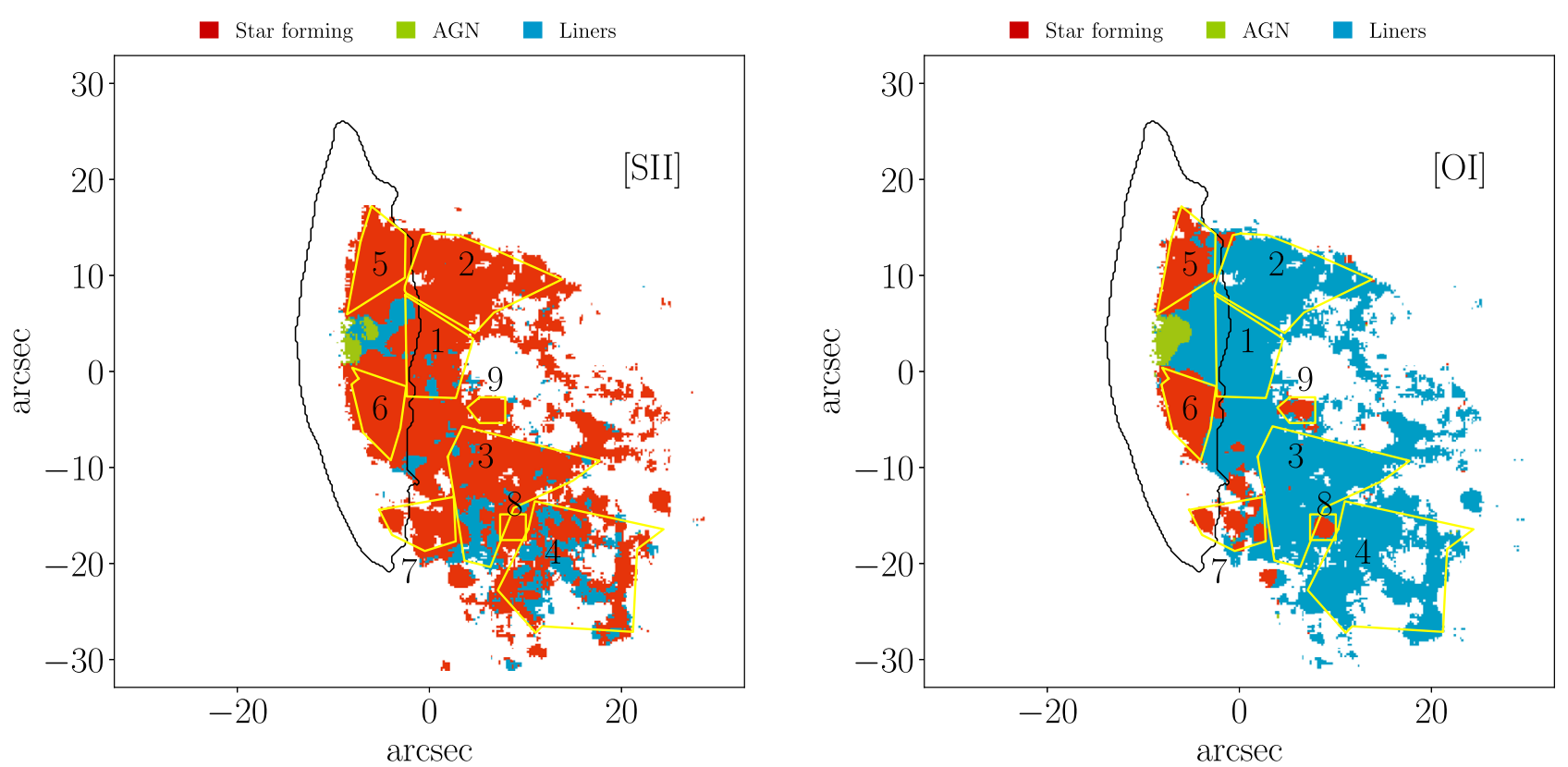

Figure 2. Ionization mechanism map according to (left) the [O III] 5007/H $\beta$ vs. [S II] 6717,6731/H $\alpha$ and (right) [O III] 5007/H $\beta$ vs. [O I] 6300/H $\alpha$ diagnostic diagrams. Red $=\mathrm{SF}$, green $=\mathrm{AGN}$, and blue $=$ LINER-like emission. The yellow polygons define the regions that are studied in Section 5.3. The black contour shows the stellar disk as in Figure 1.

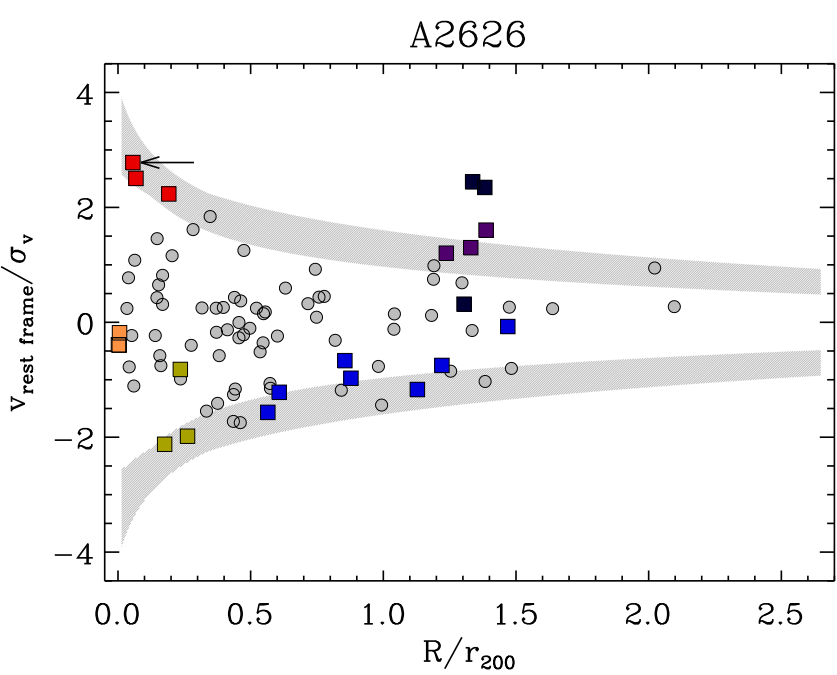

Figure 3. Distribution of cluster members in projected phase space. Projected clustercentric distances $R$ and rest-frame line-of-sight velocities $v_{r f}$ are in units of the cluster virial radius and velocity dispersion, respectively (both taken from Biviano et al. 2017). Galaxies belonging to different substructures are represented by colored squares, each color defining a different substructure. The arrow points to JW100, which belongs to the substructure that also includes the other two galaxies identified by red squares. The gray shaded regions represent the escape velocity curves with $1 \sigma$ uncertainties, assuming a Navarro et al. (1996) profile and Tiret et al. (2007) velocity anisotropy profile. The width of these regions takes into account $1 \sigma$ uncertainties in the mass profile parameters $r_{200}$ and $r_{-2}$ from Biviano et al. (2017) and allowing the

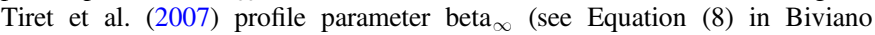
et al. 2013) to vary from zero (isotropic orbits) to 0.75 (radial orbits).

\section{Results of the X-Ray Analysis}

While the analyses of the MUSE and ALMA data are presented elsewhere (Poggianti et al. 2017a; Poggianti et al. 2019; Moretti et al. 2019), in this section, we describe the first detailed analysis of the X-ray data of JW100.

\subsection{Detection of Point Sources}

We ran WAVDETECT on the two Chandra observations separately. There are only two point sources detected in the galaxy area, whose position and source counts are listed in Table 2 . They are readily seen in excess to the galaxy diffuse emission in Figure 4, in which the soft, medium, and hard Chandra bands are shown. The contrast with the diffuse emission increases going from the soft to the hard band.

The first source is positionally coincident with the center of the galaxy and consistent with the AGN reported in Radovich et al. (2019). The second source is just outside the optical extent of the stellar disk but within the X-ray tail. We remove these sources for the analysis of the X-ray diffuse emission described below.

\subsection{Spectral Analysis of the Galaxy}

We performed a spectroscopic analysis of the Chandra data with XSPEC v12.10 (Arnaud 1996). We defined the region of interest of the spectral analysis, i.e., the galaxy, based on the MUSE observation to include the disk and stripped tail. Then we defined a control region to study the properties of the ICM surrounding the galaxy. The ICM of A2626 has an almost spherical symmetry (Wong et al. 2008; Ignesti et al. 2018; Kadam et al. 2019); thus, we expect all of the thermal plasma at the same clustercentric distance of the galaxy to have similar properties. ${ }^{24}$ To maximize the photon statistics, we used as a control region a ring-shaped sector at the same distance as JW100. The galaxy and control regions are shown in Figure 5.

We extracted a spectrum in each of the two regions using the CIAO task specextract and then binned to give at least 25 counts in each energy bin. Similarly, we extracted the background spectrum from the Blank-Sky files in the same

\footnotetext{
${ }^{24}$ We neglect the increase in temperature due to the galactic Mach cone, because in projection, it will be only a minor effect.
} 
Table 2

Properties of the Point Sources

\begin{tabular}{|c|c|c|c|c|c|c|c|c|}
\hline Source & ObsID & R.A.(J2000) & Decl.(J2000) & Net Counts & Net $(0.3-2 \mathrm{keV})$ & Net $(2-10 \mathrm{keV})$ & $\begin{array}{l}f_{X}^{(0.5-2 \mathrm{keV})} \quad f_{X}^{(2-10 \mathrm{keV})} \\
\quad \times 10^{-15} \mathrm{erg} \mathrm{cm}^{-2} \mathrm{~s}^{-1}\end{array}$ & $\begin{aligned} & L_{X}^{(0.5-10 \mathrm{keV})} \\
\times & 10^{40} \mathrm{erg} \mathrm{s}^{-1}\end{aligned}$ \\
\hline$\overline{\mathrm{AGN}}$ & 3192 & $23: 36: 25.031$ & $+21: 09: 02.53$ & $43.8 \pm 7.3$ & $22.9 \pm 5.3$ & $21.7 \pm 4.8$ & & \\
\hline $\mathrm{AGN}$ & 16136 & & & $185.5 \pm 14.93$ & $99.9 \pm 10.8$ & $81.7 \pm 9.4$ & 32.7 & 23.8 \\
\hline ULX? & 3192 & $23: 36: 24.592$ & $+21: 08: 47.65$ & $35.8 \pm 6.7$ & $28.9 \pm 5.8$ & $9.7 \pm 3.3$ & & \\
\hline ULX? & 16136 & & & $130.5 \pm 13.0$ & $85.9 \pm 10.1$ & $42.7 \pm 7.0$ & 6.6 & 7.2 \\
\hline
\end{tabular}

Note. Positions, net counts, unabsorbed fluxes, and luminosity. See Section 6 for details on the spectral shapes used.

regions. The point sources were removed or masked (radius 1".8) during the spectrum extraction.

Spectra have been extracted separately from the two observations generating independent response matrices and then, after background subtraction, fitted jointly in the energy range $0.5-7 \mathrm{keV}$.

The control region spectrum was fitted with an absorbed thermal model (phabs*apec), and the results are reported in Table 3 . We measure $k T=3.5 \pm 0.1 \mathrm{keV}$, a metallicity $Z=0.36 \pm 0.04$ solar, and an electron density $n_{e}=3.2 \cdot 10^{-3} \mathrm{~cm}^{-3}$ that corresponds to an ICM density $\rho_{\text {ICM }}$ of $5.8 \cdot 10^{-27} \mathrm{~g} \mathrm{~cm}^{-3}$. The properties of the ICM we derive here are in agreement with previous results by Ignesti et al. (2018) and Kadam et al. (2019).

As a first result, we ruled out that the ICM emission alone can reproduce the observed emission from JW100 because a single-temperature model (apec model) is not a good fit, as shown by the final statistics presented in Table 4 ( $\chi^{2}=173.43$, dof $=96$ ).

Therefore, we modeled the spectrum extracted in the galaxy region as the combination of two components. To model the cluster emission along the line of sight, we used the absorbed, thermal, single-temperature component (apec) described above whose properties were fixed to those of the ICM measured in the control region (Table 3). Then, to model the galactic emission itself, we adopted either another singletemperature apec model or a multiphase, multitemperature model, where the plasma emission measure $\mathrm{EM}=\int n_{e} n_{\mathrm{H}} d V$; i.e., the normalization of the bolometric power emitted as thermal radiation scales with the temperature as EM $\propto T^{\alpha}$, and the temperature has an upper limit $T_{\max }$ (hereafter the cemekl model; Singh 1996).

The former is a simplified model where the galactic medium is a plasma emitting at a single temperature that is different from that of the local ICM. The latter is appropriate for a scenario in which the galactic X-ray emission comes from a multitemperature plasma that could be produced by the mixing of the hot ICM and the cold interstellar medium (ISM) triggered by the ISM stripping, thermal conduction heating, or shock heating. In this case, we may expect the temperature of the emitting plasma to range from the temperature of the ICM to the temperature of the ISM. The photon statistics was not sufficient to obtain a solid estimate of the metallicity of the plasma, so we fixed it at the solar value, which is the metallicity of the stripped gas measured from the MUSE data.

We report the results of the fits in Table 4. With the double apec model $\left(\chi^{2}=93.58\right.$, dof $\left.=95\right)$, we recover a temperature of $0.82_{-0.05}^{+0.16} \mathrm{keV}$ for the galactic component, which is lower than the ICM. In the apec+cemekl model, we fitted the data first by setting the $\mathrm{T}_{\max }$ parameter to match the temperature of the $\operatorname{ICM}\left(\chi^{2}=93.84\right.$, dof $\left.=95\right)$, then by letting it free $\left(\chi^{2}=87.18\right.$, dof $\left.=94\right)$. In the second case, we recovered an upper limit of the temperature $\mathrm{T}_{\max }=1.2_{-0.26}^{+0.50} \mathrm{keV}$, which is lower than the ICM temperature. The two models (double apec versus apec+cemekl) are statistically indistinguishable, and they fit the observations equally well.

For each model, we measured the unabsorbed X-ray luminosity in the $0.5-2.0,0.5-10.0$, and $0.3-10 \mathrm{keV}$ bands associated with the galactic spectral component, listed in Table 4.

These findings will be discussed in Section 5.3.

\subsection{Search for the Bow Shock}

With respect to the cluster, JW100 has a line-of-sight velocity of $1807 \mathrm{~km} \mathrm{~s}^{-1}$, and, based on the orientation of the $\mathrm{H} \alpha$ tail, we expect the total velocity to also have a significant transversal component. From the values of the thermal properties of the ICM measured in the control region, we estimate a local sound velocity $c_{\mathrm{s}} \simeq 1.5 \cdot 10^{4} T_{\mathrm{ICM}}^{1 / 2} \simeq 960 \mathrm{~km} \mathrm{~s}^{-1}$. Therefore, the galaxy is moving supersonically (with a tentative lower limit for the galaxy Mach number $\mathcal{M} \simeq 2$ ), and, thus, we may expect to observe two discontinuities in front of it: the leading edge of the shock, i.e., the bow shock, and the contact discontinuity that drives this shock. Measuring the jump temperature across the shock front could give us an independent measure of the galaxy Mach number and thus of its velocity with respect to the ICM. We note that bow shocks in front of jellyfish cluster galaxies have never been observed (but see Rasmussen et al. 2006 for the temperature jump in NGC 2276 in a galaxy group).

We performed a morphological analysis to search for a brightness discontinuity in front of the infalling galaxy by adopting several geometries, finding indications of a surface brightness jump at $\sim 6^{\prime \prime} \sim 6 \mathrm{kpc}$ from the galaxy with a significance of $2 \sigma$ (Figure 6). To have a spectroscopic confirmation, we further measured the temperature profile across the surface brightness jump. We extracted the spectra in the $0.5-7.0 \mathrm{keV}$ band in the supposedly post-shock (orange) and pre-shock (blue) regions across the brightness edge (Figure 6), and we collected 1700 and 650 photons in the outer and inner sectors, respectively. Our spectral results may suggest a temperature jump at the shock front $\left(k T_{\text {pre }}=\right.$ $\left.4.33_{0.20}^{+0.30} \mathrm{keV}, k T_{\text {post }}=4.88_{-0.39}^{+0.56} \mathrm{keV}\right)$, although given the uncertainties, the pre- and post-shock regions are still consistent with being isothermal. From this analysis, we therefore conclude that the Chandra data can neither confirm nor deny the existence of the shock front. This may be caused by the combination of the low data statistics and the complex morphology of the shock, as suggested by the $\mathrm{H} \alpha$ surface brightness distribution. We note that with the expected Mach number $\mathcal{M} \gtrsim 2$, we would have a physical temperature jump 

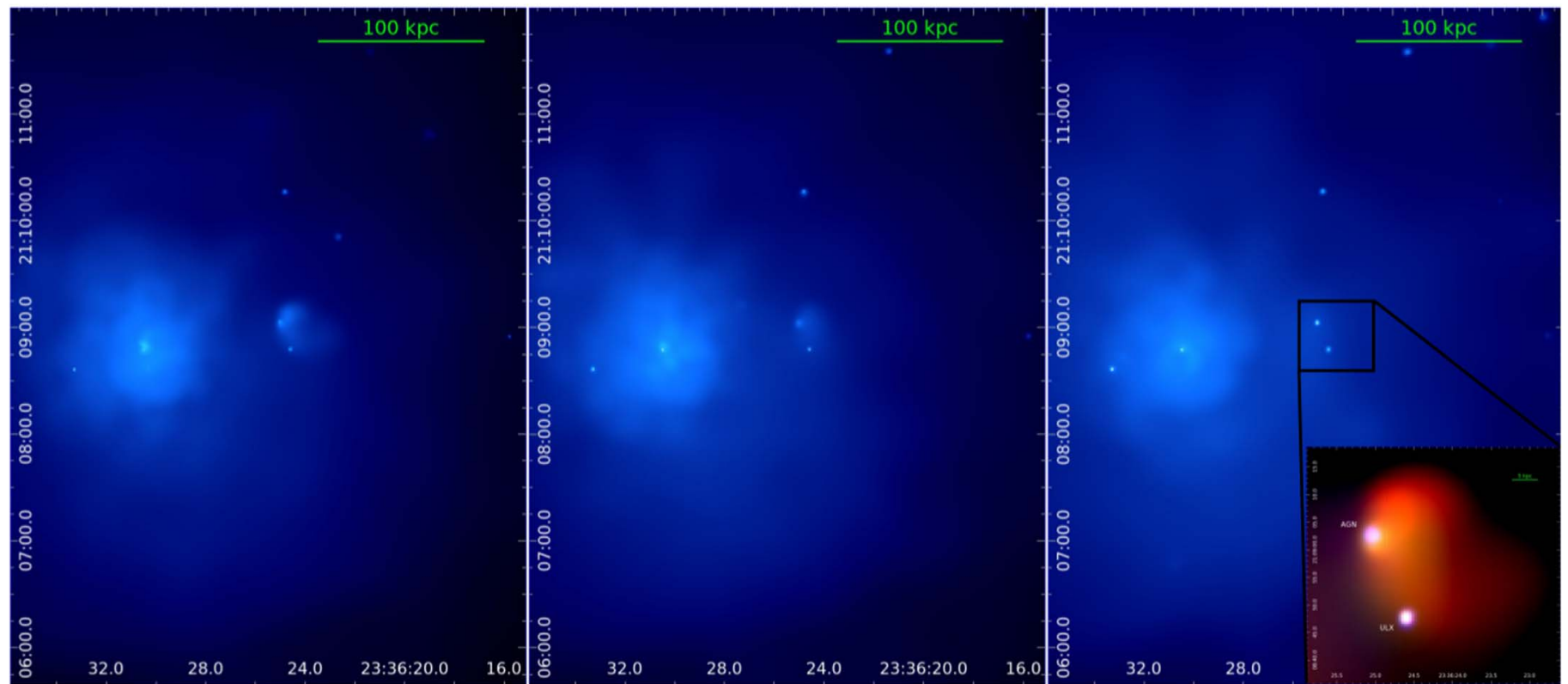

Figure 4. Cluster-scale images showing the three X-ray bands (soft 0.5-1.2 (left), medium 1.2-2.0 (middle), and hard 2.0-7.0 (right) keV) of the field of JW100, smoothed with csmooth in CIAO. The inset in the right panel is an RGB (red $=0.5-1.2$, green $=1.2-2.0$, and blue $=2.0-7.0 \mathrm{keV}$ ) smoothed zoom on the galaxy where the two point sources (AGN and ULX candidate) are clearly visible.

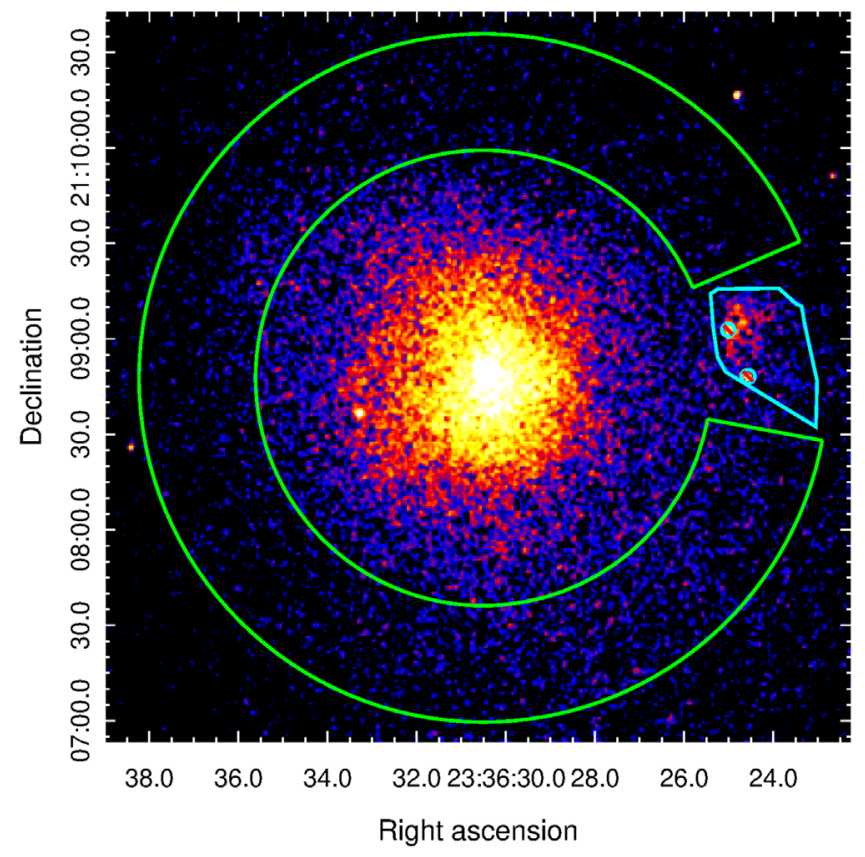

Figure 5. Background-subtracted, exposure-corrected Chandra image of A2626 in the $0.5-2.0 \mathrm{keV}$ band smoothed with a 1".5 Gaussian beam, with the galaxy (cyan) and control (green) regions highlighted. The two small circles within the JW100 region are the two point sources discussed extensively in Section 6.

around 2. However, projection effects would significantly decrease the jump and smear out the discontinuity.

\section{Results: The Spatial Distribution and Physical Origin of the Emission at Different Wavelengths}

In this section, we analyze the spatial distribution of the emission at different wavelengths. Figure 7 presents the $\mathrm{H} \alpha$, NUV, $\mathrm{CO}(2-1)$, X-ray $(0.5-2.0 \mathrm{keV})$, and radio continuum $1.4 \mathrm{GHz}$ images. At all wavelengths, JW100 displays an extraplanar tail to the west of the disk. Analyzing the morphology and characteristics of the emission at different wavelengths, in the following, we investigate the physical origin of the multiphase tail.

\section{1. $\mathrm{H \alpha}, \mathrm{CO}$, and $U V$}

The most extended tail observed is the $\mathrm{H} \alpha$ one, reaching out to at least $50 \mathrm{kpc}$ outside of the galaxy stellar disk, where the MUSE field of view ends. Figure 7 clearly shows that the $\mathrm{H} \alpha$ emission is composed of bright clumps embedded in diffuse emission (see also Poggianti et al. 2019). Within the disk, the $\mathrm{H} \alpha$-emitting gas is only present in the western half (i.e., downstream) of the disk and out to about $14 \mathrm{kpc}$ from the galaxy center along the disk major axis. At the eastern edge of the $\mathrm{H} \alpha$ emission, the gas is compressed by the ram pressure (see also contours in the other panels of Figure 7). ${ }^{25}$ The outer regions of the disk $(r>14 \mathrm{kpc}$ north and south of the galaxy center) and all of the eastern projected side have already been stripped of gas by ram pressure.

The $\mathrm{H} \alpha$ velocity map (Figure 1 in Poggianti et al. 2017b; not shown here) indicates that, as is typical of jellyfish galaxies, the stripped gas maintains the disk rotation quite coherently downstream and suggests that in the plane of the sky, the galaxy is moving with respect to the ICM $\sim 45^{\circ}$ northeast.

The ionization source of the bright $\mathrm{H} \alpha$ clumps is photoionization by young massive stars and thus SF taking place during the last $\leqslant 10^{7} \mathrm{yr}$, as is consistently found by both the [S II] and [O I] diagnostic diagrams (Figure 2). The starforming clumps are mostly located in the southern part of the tail.

The origin of the ionization of the diffuse component is instead more uncertain, as SF dominates according to the [S II] diagram, while LINER-like emission dominates for the [O I]

\footnotetext{
${ }^{25}$ While shear can also remove gas from a galaxy, compression is more likely than shear to cause an enhancement in $\mathrm{H} \alpha$ emission. Also, as this galaxy has a significant velocity component moving toward the east, the ICM stagnation point is likely on the eastern side, reducing the strength of the shear instabilities.
} 
Table 3

Results of the X-Ray Spectral Analysis in the Control Region

\begin{tabular}{lcccc}
\hline \hline ObsID & Exposure Time (s) & Bkg. Exp. Time (s) & Total Counts (counts) & Net Rate (counts s ${ }^{-1}$ ) \\
\hline 16136 & $1.047 \mathrm{e}+05$ & $6.725 \mathrm{e}+05$ & 29,992 & $0.266(92.7 \%$ total) \\
3192 & $2.368 \mathrm{e}+04$ & $4.615 \mathrm{e}+05$ & 8445 & $0.337(94.4 \%$ total) \\
\hline \multicolumn{2}{c}{ Model } & & Parameters & $\chi^{2}$, dof, $\chi_{R}^{2}$ \\
\hline \multicolumn{2}{c}{ phabs*apec } & $k T=3.50 \pm 0.10 \mathrm{keV}, Z=0.36 \pm 0.04$ & $499.02,456,1.0943$
\end{tabular}

diagram (Figure 2). These apparently contrasting conclusions probably indicate that both SF and another source of ionization contribute to the diffuse line emission, but assessing the relative contribution of the two processes is very hard based on diagnostic diagrams. In the hypothesis that the stellar photons ionizing the diffuse gas are those escaped from the $\mathrm{H}$ II regions within the clumps, Poggianti et al. (2019) derived for JW100 an escape fraction of $52 \%$, by far the highest in the GASP sample whose average is $18 \%$. Moreover, JW100 is the galaxy that most deviates from the anticorrelation between SFR in the tail and the fraction of tail $\mathrm{H} \alpha$ emission that is diffuse (Figure 12 in Poggianti et al. 2019), demonstrating an excess of tail diffuse emission compared to clump emission. This is all consistent with the fact that the JW100 tail might have an unusually high contribution from sources of ionization other than in situ SF. This also agrees with the fact that, as visually assessed from Figure 7, significant UV emission is lacking in the regions of diffuse $\mathrm{H} \alpha$ emission with a high $[\mathrm{O} \mathrm{I}] / \mathrm{H} \alpha$ ratio, supporting the notion that in situ SF may be lacking in such areas, though we cannot exclude that fainter UV emission below our detection limit is present. A strong UV emission obscured by a large amount of dust can be excluded in the $\mathrm{H} \alpha$ diffuse emission regions, based on the moderate to low levels of dust extinction ( $A_{V}$ values typically between 1 and $0.2 \mathrm{mag}$ ) derived from the Balmer decrement map observed with MUSE (not shown).

Molecular gas, as traced by $\mathrm{CO}(2-1)$ emission, is present only in the area of the disk where $\mathrm{H} \alpha$ emission is also present (middle panels of Figure 7). Extraplanar CO complexes are also found close to the disk (within a few kpc) in the northern part of the tail and out to $\sim 30 \mathrm{kpc}$ from the disk in the southern part of the tail. A more detailed analysis of the ALMA data, both $\mathrm{CO}(2-1)$ and $\mathrm{CO}(1-0)$, and the spatially resolved SF efficiency in JW100 is presented in a separate paper (Moretti et al. 2019; see also Lee et al. 2017 for a comparison of CO, $\mathrm{H} \alpha$, and UV data of four ram pressure stripped galaxies in Virgo, though only within or just outside of the disks).

For the purposes of this paper, it is interesting to compare the $\mathrm{CO}$ emission with both the $\mathrm{H} \alpha$ emission (middle left panel) and the regions that are powered by SF according to both the [O I] and $[\mathrm{S} \mathrm{II}]$ diagram (middle right panel). Some of the $\mathrm{CO}$ complexes spatially coincide with some of the $\mathrm{H} \alpha$ clumps, but there are also $\mathrm{H} \alpha$ clumps with little or no $\mathrm{CO}$ (e.g., clump 1 in Figure 7) and $\mathrm{CO}$ complexes with only diffuse, low surface brightness $\mathrm{H} \alpha$ emission (e.g., clump 2). Moreover, not all of the $\mathrm{H} \alpha$ clumps with corresponding $\mathrm{CO}$ emission are classified as star-forming according to the [O I] diagram (e.g., clump 3).

These findings can be reasonably explained by a combination of two factors. The fact that not all $\mathrm{H} \alpha$ clumps with $\mathrm{CO}$ emission are classified as star-forming according to the [O I] diagram might be due to the coexistence of different ionization mechanisms contributing at the same location, or at least powering ionized regions superimposed along the same line of sight.

The fact that $\mathrm{CO}$ and $\mathrm{H} \alpha$ clumps do not always spatially coincide might be due instead to the evolutionary stage of the star-forming regions. Each star-forming region will go through four phases: (a) a molecular gas-only phase (no massive stars formed yet), (b) a molecular gas + ionized gas + UV light phase (massive stars have had the time to ionize the surrounding gas, and they shine in the UV, but they have not yet dispersed the remaining molecular cloud), (c) a phase with ionized gas + UV light (the molecular clouds have been destroyed, but there are still massive stars that ionize the gas and shine), and (d) a UV light-only phase. In the latter phase, the stars more massive than about $20 M_{\odot}$ have died, and the ionizing radiation of the stars left is not capable of ionizing a significant amount of gas, but the UV radiation from the most massive stars on the main sequence is still sufficient to let the region shine in the UV. This is the case if the SF occurred more than $10^{7} \mathrm{yr}$ and less than a few times $10^{8} \mathrm{yr}$ ago.

Comparing the first four panels of Figure 7 , we see this evolutionary sequence of star-forming regions going from regions like 2,3 , and 8 (CO clouds but no bright $\mathrm{H} \alpha$ clump nor UV yet), to regions like 4 and $9(\mathrm{CO}+\mathrm{H} \alpha+\mathrm{UV})$, to regions like 1 and $5(\mathrm{H} \alpha+\mathrm{UV}$, very little or no CO left), and, finally, to $\mathrm{UV}$-only regions such as region 10 in the tail and regions 6 and 7 in the outer parts of the disk, where only the UV light has remained to testify to the recently quenched SF where all gas has been stripped. This decoupling of the various phases of the SF process is similar to the decoupling observed by, e.g., Kruijssen et al. (2019), who found a decoupling of CO- and $\mathrm{H} \alpha$-dominated clumps on $\sim 100$ pc scales in NGC 300, a close, face-on star-forming disk galaxy.

Interestingly, in the tail of JW100, we see that the different phases are located according to a spatial progression going from left to right (oldest to youngest) in the southern part of the tail. This spatial progression is summarized in Figure 8, where the different stages are shown in different colors: red where only $\mathrm{CO}$ is observed, orange for $\mathrm{CO}+\mathrm{UV}+\mathrm{H} \alpha$, green for $\mathrm{UV}$ $+\mathrm{H} \alpha$, and cyan for UV-only. Here only the $\mathrm{H} \alpha$ emission due to $\mathrm{SF}$ according to the [O I] diagram has been considered. The first stage of the SF sequence (only $\mathrm{CO}$ ) is preferentially located to the right of the other colors, and the following stages are found progressively to the left. In some cases, this sequence is at least partially observed even within an individual starforming clump (e.g., clump 4; from right to left: orange, green, and cyan). This latter effect is similar to the "fireballs" observed in the tail of ESO 137-001 by Jachym et al. (2019).

The progression observed in Figure 8 thus traces the timing of SF in the tail and strongly suggests that the molecular clouds further away from the disk have not formed stars (yet). This agrees with the (counterintuitive) stellar age gradient in the tail found in some jellyfish galaxies, by which younger stellar 
Table 4

Results of the X-Ray Spectral Analysis in the Galactic Region

\begin{tabular}{|c|c|c|c|c|}
\hline ObsID & Exposure Time (s) & Bkg. Exp. Time (s) & "Total Counts (counts) & Net Rate (counts s ${ }^{-1}$ ) \\
\hline 16136 & $1.047 \mathrm{e}+05$ & $6.725 \mathrm{e}+05$ & 2502 & $0.0228(95.6 \%$ total $)$ \\
\hline 3192 & $2.368 \mathrm{e}+04$ & $4.615 \mathrm{e}+05$ & 632 & $0.0260(97.4 \%$ total $)$ \\
\hline \multicolumn{2}{|c|}{ Model } & \multicolumn{2}{|c|}{ Parameters } & $\chi^{2}$, dof, $\chi_{R}^{2}$ \\
\hline \multicolumn{2}{|c|}{ phabs*apec } & \multicolumn{2}{|c|}{$k T=1.99 \pm 0.14 \mathrm{keV}, Z=0.11 \pm 0.05$} & $173.43,96,1.8066$ \\
\hline \multicolumn{2}{|c|}{ phabs* (apec+apec) } & \multicolumn{2}{|c|}{$\begin{array}{c}k T=0.82_{-0.05}^{+0.14} \mathrm{keV},(Z=1.00 \text { fixed }) \\
0.5-2.0=1.99 \mathrm{e} 41 \mathrm{erg} \mathrm{s}^{-1}, L_{0.5-10.0}=2.08 e 41 \mathrm{erg} \mathrm{s}^{-1} \\
L_{0.3-10.0}=2.21 \mathrm{e} 41 \mathrm{erg} \mathrm{s}^{-1}\end{array}$} & $93.58,95,0.9851$ \\
\hline \multicolumn{2}{|c|}{ phabs* (apec+cemekl) } & \multicolumn{2}{|c|}{$\begin{array}{c}\left(k T_{\max }=3.49 \mathrm{keV} \text { fixed }\right),(Z=1.00 \text { fixed }), \alpha=0.88_{-0.32}^{+0.31} \\
L_{0.5-2.0}=3.40 \mathrm{e} 41 \mathrm{erg} \mathrm{s}^{-1}, L_{0.5-10.0}=4.54 \mathrm{e} 41 \mathrm{erg} \mathrm{s}^{-1} \\
L_{0.3-10.0}=5.00 \mathrm{e} 41 \mathrm{erg} \mathrm{s}^{-1}\end{array}$} & $93.84,95,0.9878$ \\
\hline \multicolumn{2}{|c|}{ phabs* (apec+cemekl) } & \multicolumn{2}{|c|}{$\begin{array}{c}k T_{\max }=1.20_{-0.26}^{+0.51} \mathrm{keV},(Z=1.00 \text { fixed }), \alpha=2.07_{-0.98}^{+3.32} \\
L_{0.5-2.0}=2.31 \mathrm{e} 41 \mathrm{erg} \mathrm{s}^{-1}, L_{0.5-10.0}=2.47 \mathrm{e} 41 \mathrm{erg} \mathrm{s}^{-1} \\
L_{0.3-10.0}=2.68 \mathrm{e} 41 \mathrm{erg} \mathrm{s}^{-1}\end{array}$} & $87.36,94,0.9293$ \\
\hline
\end{tabular}

clumps are found further away from the disk (e.g., IC 3418, Kenney et al. 2004; Fumagalli et al. 2011; RB 199, Yoshida et al. 2008; JO 201, Bellhouse et al. 2019). Hydrodynamical simulations predict very recent SF preferentially further out in the tail than closer into the disk for some infalling angles (see, e.g., Figure 1 in Roediger et al. 2014 for a face-on infall). A detailed comparison with simulations is beyond the scope of this paper and will be the subject of future work.

The recently quenched SF occurring where the gas has been totally stripped is testified to not only by the UV emission but also by the strong Balmer lines in absorption (accompanied by the lack of emission lines) in the MUSE spectra. As an example, the spectra of regions 6 and 7 shown in Figure 9 display the strong Balmer lines typical of post-star-forming and post-starburst regions (Poggianti et al. 1999). The $\mathrm{H} \beta$ restframe equivalent widths of these spectra are 6.7 and $6.6 \AA$, respectively, which can only arise from stellar populations less than 1 Gyr old whose spectra are dominated by A-type stars.

To summarize, the observables that are more closely linked with ongoing/recent SF (the gas ionized by SF, the molecular gas, and the UV emission) all point to the southern half of the tail as the location of in situ SF extending much further away from the disk than in the northern part. Although overall, these three observables depict a similar picture, the exact location of $\mathrm{H} \alpha$, CO, and UV emission does not always coincide on small scales. This mismatch can be ascribed to the different SF stages and timescales traced by $\mathrm{H} \alpha, \mathrm{CO}$, and $\mathrm{UV}$ (thus, an evolutionary sequence within star-forming regions), in some cases combined with the contribution of an additional source of gas ionization that gives rise to peculiar optical line ratios observed with MUSE, such as shock heating, thermal conduction from the ICM, or mixing of the ISM and ICM (see Section 5.3).

\subsection{Radio Continuum}

Although care should be taken when comparing images at different resolutions (given the amount of tapering required to better map the extended/diffuse radio structure, the resolution of the $1.4 \mathrm{GHz}$ map is a factor of $\sim 3$ worse than that of the other images, which is about $1^{\prime \prime}$ ), we note that the $1.4 \mathrm{GHz}$ emission appears to have a different spatial distribution from all other wavelengths (bottom right panel of Figure 7).

In the disk, it globally coincides with the location of the $\mathrm{H} \alpha$ and $\mathrm{CO}$ (and X-ray) emission, though with a slight extension to the east of the ram pressure edge, in correspondence to the galaxy center, most likely due to the radio continuum emission from the AGN, whose pointlike morphology is clearly detected in the high-resolution images at $5 \mathrm{GHz}$ presented in Gitti (2013; Figure 4) and Ignesti et al. (2017; Figure 1).

Outside of the disk, extended $1.4 \mathrm{GHz}$ emission is detected. We note that such emission is indeed diffuse, as it is not evident in the $1.4 \mathrm{GHz}$ maps, which have a synthesized beam about or slightly higher than $1^{\prime \prime}$ (the untapered reconstructed images; see Figures 1 and 2 of Gitti 2013). In particular, a northern radio continuum tail extends approximately as much as the NUV and X-ray emission. There is also a radio south tail that covers patchy areas with no $\mathrm{CO}$, no bright $\mathrm{H} \alpha$ knots (except for one knot in the southeast), and almost no X-ray detected. The radio continuum emission, therefore, is generally present in regions that are lacking ongoing clumpy SF/high molecular gas content. There is instead at least some UV emission in the majority of radio-emitting tail regions, except for the southwest radio clump.

In order to probe the thermal or nonthermal origin of the radio emission, we estimated the spectral index of the tail between 1.4 and $5.5 \mathrm{GHz}$ using the VLA maps presented in Ignesti et al. (2017; Figure 1). At $5.5 \mathrm{GHz}$, we do not observe diffuse radio emission in the tail, so by considering the $3 \sigma$ level, we could estimate only a lower limit for the spectral index $\alpha>0.5$ (in this work, the spectral index $\alpha$ is defined as $S \propto \nu^{-\alpha}$, where $S$ is the radio flux and $\nu$ is the frequency). Tabatabaei et al. (2017) fitted the $1-10 \mathrm{GHz}$ spectral energy distribution of nearby galaxies using a Bayesian Markov Chain Monte Carlo technique in order to disentangle the thermal and nonthermal contributions to the radio emission. These authors measured a total spectral index (combination of thermal and nonthermal components) ranging from 0.5 to 1.0 . Then, by assuming $\alpha=0.1$ for the thermal radio emission, they estimated a mean thermal fraction at $1.4 \mathrm{GHz}$ of $10 \%-13 \%$. 

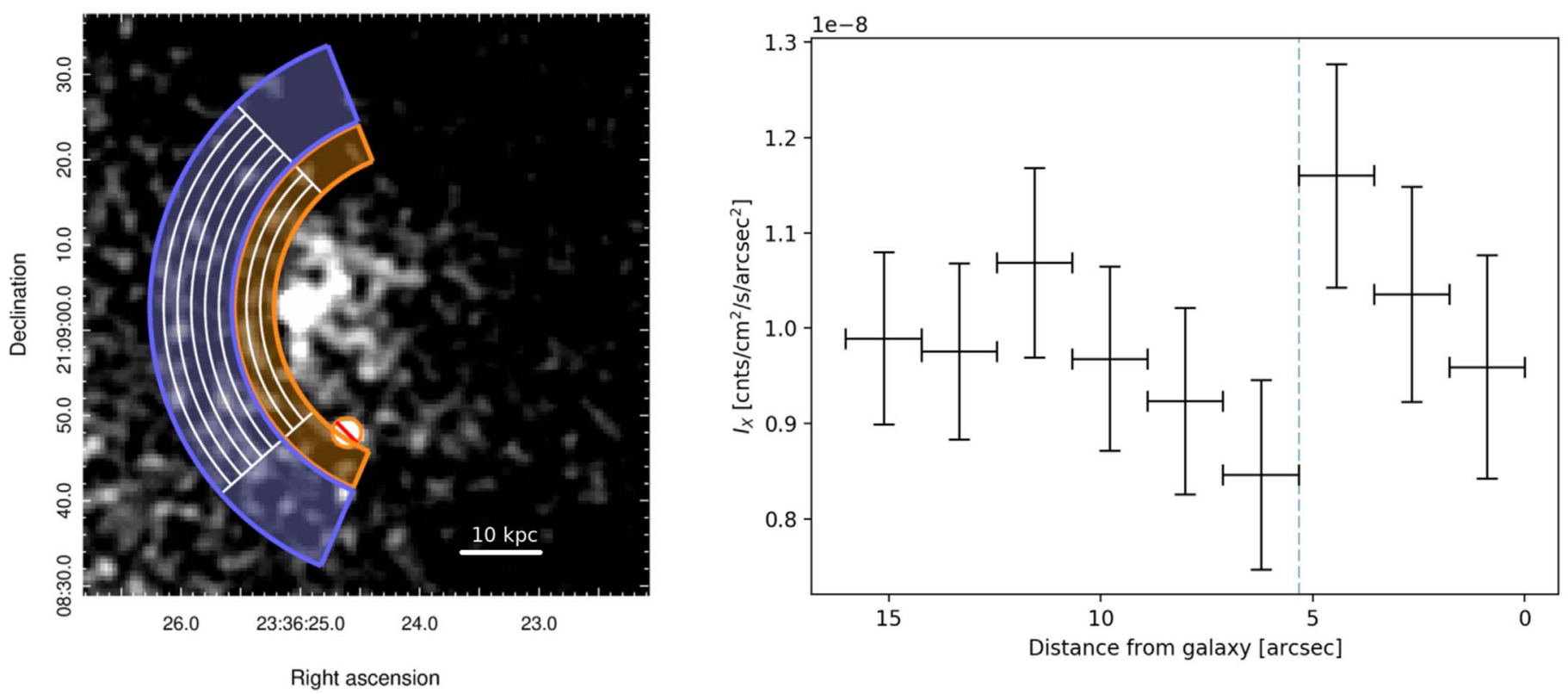

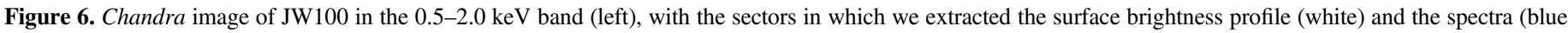

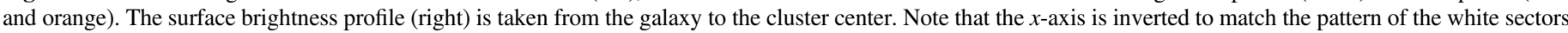
in the left panel. The vertical dashed line points out the location of the promising discontinuity, which is located between the orange and blue sectors.

Therefore, from our estimated lower limit, we may conclude that the fraction of nonthermal contribution to the total radio emission from the tail of JW100 is more than 90\%, thus indicating that we are mainly observing synchrotron emission of relativistic electrons diffused in the tail of JW100. The spectral index of the disk is $\sim 0.6-0.7$, and it may be contaminated by the AGN.

The origin of relativistic electrons and magnetic field in the stripped tail is uncertain. The expected $L_{1.4 \mathrm{GHz}}$ associated with the SFR derived from MUSE and converted from a Salpeter to a Chabrier IMF using the Bell (2003) calibration $\mathrm{SFR}=3.25 \times 10^{-22} L_{1.4 \mathrm{GHz}}\left(\mathrm{W} \mathrm{Hz}^{-1}\right)$ is $2.5 \cdot 10^{21}$ and $9.8 \cdot 10^{21} \mathrm{~W} \mathrm{~Hz}^{-1}$ for the tail and disk, respectively. These values are consistent with the observed $L_{1.4 \mathrm{GHz}}$ (Table 5), thus suggesting that core-collapse supernovae may be the dominant source of relativistic electrons. However, we cannot exclude the contribution of other sources, such as stripping of relativistic electrons from the galaxy due to ICM winds (Murphy et al. 2009). Regarding the magnetic field in the tail, it may be the combination of the ISM magnetic field following the stripped plasma and the ICM magnetic field, but with the present data, we could not perform a study of polarized emission to disentangle them. The magnetic field measurement in a GASP jellyfish tail will be presented in A. Mueller et al. (2019, in preparation). Interestingly, the radio emission in the tail of JW100 on small scales often does not spatially coincide with the regions of ongoing SF (the $\mathrm{CO}$ and brightest $\mathrm{H} \alpha$ clumps). Assuming that most of the relativistic electrons derive from the SFR, this might be due to timescale issues. During their lifetime, the accelerated electrons can travel large distances, and/or at the location of the youngest star-forming regions, it is possible that there are not yet any powerful accelerating sources of electrons; i.e., there have been no supernova explosions.

\subsection{X-Ray}

In contrast to the SF tracers described in Section 5.1, the $\mathrm{X}$-ray extraplanar emission is much more conspicuous in the northern half of the tail than in the southern half, with a ratio in the $0.5-2.0 \mathrm{keV}$ band between north and south of $\sim 4$ in luminosity and $\sim 6.5$ in surface brightness (see regions $1-2$ versus 3-4 in Figure 10). The southern tail contains a bright point source, a candidate ULX, that is located just outside of the stellar disk, which will be discussed in detail in Section 6 .

Interestingly, the sharp eastern edges of X-ray, $\mathrm{H} \alpha$, and $\mathrm{CO}$ emission in the disk coincide along a vertical line where all three gas phases are compressed by the ram pressure. The extension and location of all three gas phases in the disk also coincide, as they are only found on the western side of the central $14 \mathrm{kpc}$, while all outer disk regions and the eastern side of the disk are devoid of all gas phases.

The galaxy JW100 shows its own extended X-ray emission, on a scale $\geqslant a$ dozen $\mathrm{kpc}$, standing above the emission of the cluster (see spectral analysis in Section 4.2). This emission might have different origins: (a) SF within the tail; (b) a stripped hot galaxy halo; or (c) heating of the cold ISM by either shocks, thermal conduction, or mixing with the ICM. We do not see any anisotropic features in the X-ray emission that may suggest the presence of jets powered by AGNs; also unlikely is a contribution to the extended X-ray emission from the nuclear outflow revealed by optical emission lines, considering the low-mass rate (see Sections 2 and 6).

(a) Let us start by considering the first hypothesis. In the presence of SF, the dominant contribution to the X-ray emission is expected from high-mass X-ray binaries (HMXBs), which have a lifetime of a few $10^{7} \mathrm{yr}$ and dominate over the emission of low-mass X-ray binaries when there is vigorous ongoing SF. A smaller contribution arises from the hot ISM ionized by supernovae and massive stars. Each of these contributions, as well as the sum of the two, correlate well with 

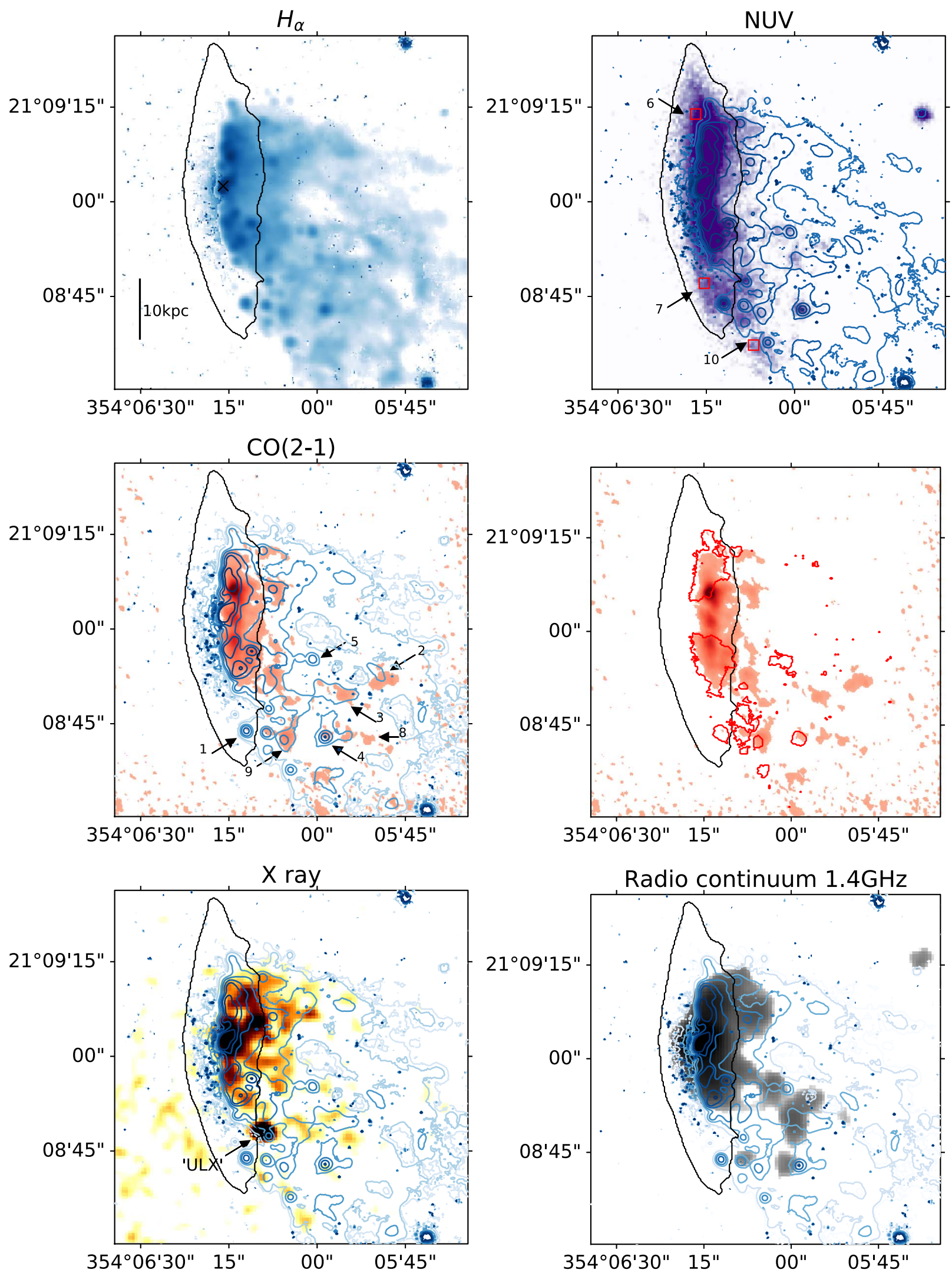

Figure 7. The JW100 multiwavelength tails. Images displayed are (top panels) the MUSE H $\alpha$ (1" resolution) and UVIT NUV (1". 2), (middle) ALMA CO(2-1) (1"), and (bottom) Chandra X-ray ( $\left({ }^{\prime \prime} 5\right)$ and VLA $1.4 \mathrm{GHz}\left(3{ }^{\prime \prime} 8 \times 3\right.$." 4$)$. Overlaid blue contours are the MUSE H $\alpha$ emission. The red contours in the middle right panel outline the star-forming regions in Figure 2. The stellar disk region is shown by the black contours. The positions marked with arrows and numbers are discussed in the text (Section 4.1). The cross in the top left panel identifies the galaxy center, defined as the centroid of the continuum (stellar) emission underlying the H $\alpha$ line from MUSE. 


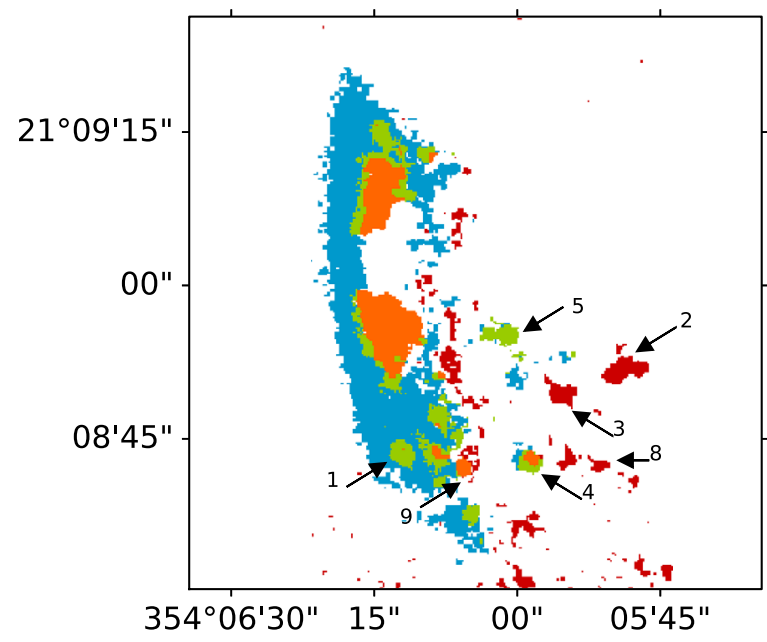

Figure 8. The SF sequence created by ram pressure: only $\mathrm{CO}$ (red), $\mathrm{CO}+\mathrm{SF}-$ powered $\mathrm{H} \alpha+\mathrm{UV}$ (orange), SF-powered $\mathrm{H} \alpha+\mathrm{UV}$ (green), and only UV (cyan). The regions with SF-powered $\mathrm{H} \alpha$ are also star-forming according to the [O I] line (right panel in Figure 2). Numbers are the same as in the middle left panel of Figure 7.

other SFR indicators (Ranalli et al. 2003; Mineo et al. 2012a, 2012b, 2014).

To test whether the observed X-ray luminosity of JW100 is compatible with the SFR measured from the optical lines, we use the $L_{X}-$ SFR calibration from Mineo et al. (2014) converted from a Salpeter to a Chabrier IMF and from 0.5-8 to $0.5-10 \mathrm{keV}$ assuming a factor of 1.11: $\mathrm{SFR}=1.32 \times$ $10^{-40} L_{X(0.5-10)} \mathrm{erg} \mathrm{s}^{-1}$. With this calibration, the total X-ray luminosity of JW100 listed in Table 4 corresponds to $\mathrm{SFR}=28 \pm 5$ (model apec+apec), $60 \pm 11$ (model apec + cemekl with $T_{\max }$ fixed), or $33 \pm 6$ (model apec+cemekl without $T_{\max }$ fixed) $M_{\odot} \mathrm{yr}^{-1}$. We remind the reader that X-ray point sources (AGNs and candidate ULXs) have already been excluded from the calculation of the X-ray luminosities. Even excluding the region of the disk where the X-ray and $\mathrm{H} \alpha$ contours are compressed by ram pressure (identified from Figure 7), where the X-ray luminosity could be boosted, the derived SFR would still be very high, ranging between $21 \pm 4$ and $46 \pm 9 M_{\odot} \mathrm{yr}^{-1}$. In the range $0.5-10 \mathrm{keV}, \sim 50 \%$ of the counts come from the tail, and assuming the shape of the spectrum in the tail is similar to the total one, this should correspond to a tail SFR between $\sim 14 \pm 3$ and $\sim 30 \pm$ $7 M_{\odot} \mathrm{yr}^{-1}$, depending on the X-ray model adopted.

The X-ray-based SFR values are much higher than those measured from the dust- and absorption-corrected $\mathrm{H} \alpha$ luminosity ( $4 M_{\odot} \mathrm{yr}^{-1}$ total, $\sim 1 M_{\odot} \mathrm{yr}^{-1}$ in the tail; see Section 2 ), even under the most generous assumptions (using all of the regions powered by SF according to the [S II] diagram). The scatter in the $L_{X}-\mathrm{SFR}$ relation in Mineo et al. (2014) is not able to account for the low $\mathrm{H} \alpha$-based SFRs, which are a factor between 6 and 30 lower than the X-ray-based values. Using the $0.5-2 \mathrm{keV} L_{X}-\mathrm{SFR}$ relation from Ranalli et al. (2003) transformed from a Salpeter to a Chabrier IMF yields a slightly lower X-ray-based SFR (between 14 and $24 \sim 1 M_{\odot} \mathrm{yr}^{-1}$ ) than with the Mineo calibration, but these values are still higher than the $\mathrm{H} \alpha$-based SFR by a factor between 4 and 6 .

It is interesting to note that comparing the total X-ray emission (having excluded the AGN; Table 4) and the $1.4 \mathrm{GHz}$ emission (Table 5), the X-ray emission of JW100 is more than an order of magnitude too high for its radio continuum emission, according to the relation shown in Figure 1 of Mineo et al. (2014). Thus, while the radio continuum and $\mathrm{H} \alpha$ emission are consistent and can be explained with SF (see Section 5.2), the X-ray emission has a significant excess with respect to both $\mathrm{H} \alpha$ and the radio continuum.

We conclude that it is necessary to invoke an additional source of X-ray emission other than the sources linked with ongoing SF.

(b) The second hypothesis concerns the galaxy's hot, X-rayemitting halo (see Bregman et al. 2018 for a review of hot circumgalactic gas). Before being stripped, we may expect JW100 to have possessed a hot, X-ray-emitting halo that could have been stretched during the stripping, producing the observed extraplanar diffuse emission (Bekki 2009). The galaxy NGC 1961, whose stellar mass is identical to that of JW100, has a hot halo with an X-ray luminosity within 50 projected kpc, $L_{X}(0.5-2)=8.9 \pm 1.2 \times 10^{40} \mathrm{erg} \mathrm{s}^{-1}$ (Anderson et al. 2016), which is not too dissimilar from the $L_{X}(0.5-2)$ of JW100, that is, between 2 and $3.5 \times 10^{41} \mathrm{erg} \mathrm{s}^{-1}$ (Table 4). Similar X-ray luminosities $\left(L_{X}(0.5-2)=(5-10) \times 10^{40} \mathrm{erg} \mathrm{s}^{-1}\right)$ have been measured for the inner $\sim 40 \mathrm{kpc}$ hot halo of another few galaxies as massive as JW100 (Figure 4 in $\mathrm{Li}$ et al. 2016). Given the expected enhancement of X-ray luminosity in the region compressed by ram pressure, in principle, the JW100 X-ray luminosity is compatible with being the stripped hot galaxy halo.

An easy way to evaluate the importance of the hot halo stripping is to compare the ram pressure, $p_{\text {ram }} \sim 3 \times$ $10^{-10}$ dyne $\mathrm{cm}^{-2}$ (see the Appendix), to the halo thermal pressure, $p_{\text {halo }}=n_{\text {halo }} k T_{\text {halo }}$, where the halo temperature is assumed to be $T_{\text {halo }} \sim 6 \times 10^{6} \mathrm{~K}$ (Appendix). Stripping is efficient where $p_{\text {ram }} \geqslant p_{\text {halo }}$ or $n_{\text {halo }} \lesssim 0.3 \mathrm{~cm}^{-3}$. The latter condition is likely to be verified everywhere but, perhaps, the central kpc or so (e.g., Bregman et al. 2018). We also tested the hot halo stripping hypothesis with a simple model adopting a mass model for the galaxy including a stellar bulge, stellar disk, and dark matter halo (see the Appendix for details). This model finds that the hot halo will be stripped down to the galactic central kpc or less on very short timescales ( $\sim 15 \mathrm{Myr})$, which makes the stripped halo hypothesis for the observed X-ray halo highly unlikely. ${ }^{26}$

(c) Finally, the X-ray emission of JW100 can arise from heating of the ISM due to shock heating, thermal conduction from the ICM, mixing of the ISM and ICM, or cooling of the ICM onto the colder stripped ISM. The latter scenario can be explored by considering the field length for a static cold cloud immersed in a hot medium, $\lambda_{F}=\left[\kappa(T) T / n^{2} \Lambda(T)\right]^{1 / 2}$, where $\kappa$ is the thermal conductivity, $T$ and $n$ are the temperature and number density of the hot gas, and $\Lambda$ is the cooling function. This is a measure of the balance between the cloud energy gain by conduction and the energy loss by radiation (see McKee \& Begelman 1990). For a cloud size larger that $\lambda_{F}$, radiative cooling dominates, and the hot ICM condenses on the cloud. Numerically, $\lambda_{F} \sim 136 \phi_{c}^{1 / 2} T_{e, 7}^{7 / 4} n_{e}^{-1} \Lambda_{-23}^{-1 / 2} \mathrm{pc} \approx 450 \mathrm{kpc}$ for the ICM surrounding JW100 (McKee \& Begelman 1990). For this estimate, we have adopted $n \sim 3 \times 10^{-3} \mathrm{~cm}^{-3}$ and $T \sim 3.5 \mathrm{keV}$ from the X-ray analysis above. The factor $\phi_{c} \leqslant 1$, which describes the suppression of the conductivity in a magnetized plasma, has been set to $\sim 1$. The value for $\lambda_{F}$, much

\footnotetext{
26 An example of hot halo/intragroup medium stripping from an early-type galaxy could be CGCG 254-021, which is the very massive, brightest galaxy in the Z8338 cluster, in which the X-ray gas is completely detached from the galaxy (Schellenberger \& Reiprich 2015).
} 

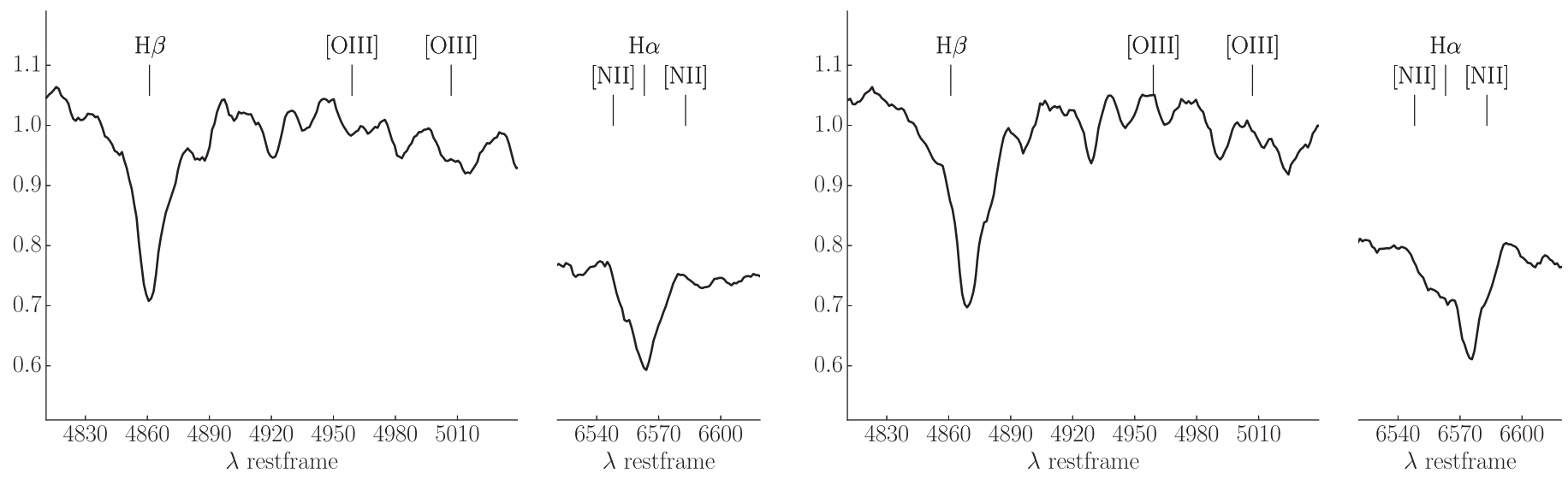

Figure 9. Spectra of regions (6) (left) and (7) (right); see red squares in Figure 7.

larger than the size of the JW100 cold ISM, makes the cooling scenario unpalatable, unless $\phi_{c} \ll 1$.

Mixing (and heating) of the stripped cold ISM with the hot ICM was invoked to explain the X-ray tail of the jellyfish ESO 137-001 in the A3627 cluster: the X-ray would arise from a warm contact surface between the ISM and the ICM that is emitting in X-rays (Sun et al. 2010).

In an oversimplified picture, the two types of X-ray spectral fitting models discussed in Section 4.2 might represent scenarios (b) and (c): the apectapec model would correspond to the stripped corona hypothesis, while the apec+cemekl model would correspond to the ISM heating hypothesis. Unfortunately, these two models are statistically indistinguishable (see $\chi^{2}$ and dof values in Table 4). Therefore, what we can conclude from our X-ray spectral modeling of JW100 (Section 4.2 and Table 4) is that the data are consistent with a multitemperature gas, and thus with the ISM heating scenario, but can neither rule out the hot halo hypothesis nor discriminate among the possible heating mechanisms.

The X-ray plasma metallicity would be a key probe to discern the models, because in the stripped hot corona scenario, we would expect the metallicity to be lower $\left(Z \simeq 0.1-0.3 Z_{\odot}\right.$; Werner et al. 2019) than that of the heated stripped gas, whose MUSE metallicity is solar and supersolar (A. Franchetto et al. 2019, in preparation). However, the existing X-ray data do not allow us to estimate the metallicity of the X-ray-emitting gas, and longer Chandra exposures are needed.

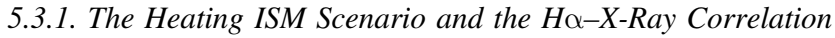

The fact that the morphology of the X-ray gas in the disk exactly follows the morphology of the $\mathrm{H} \alpha$ gas is consistent with the ISM heating scenario. We also note that in simulations with an angled wind, we find that once initial stripping has occurred, dense clouds tend to be found on the side of the tail that is more downwind (S. Tonnesen et al. 2019, in preparation). While much of the $\mathrm{X}$-ray emission is near stripped dense clouds in these simulations, there are times when the brightest X-ray emission is found near the disk and closer to the upwind side of the disk than the dense clouds, as in our JW100 observations. Moreover, in the simulations by Tonnesen et al. (2011), the authors reproduced the bright $\mathrm{H} \alpha$ and X-ray emission of the galaxy ESO 137-001 and argued that bright X-ray emission occurs when the stripped ISM is heated and mixed into a highpressure ICM (greater than $\sim 9 \times 10^{-12} \mathrm{erg} \mathrm{cm}^{-3}$ ). The JW100 ICM pressure of $\sim 3 \times 10^{-11} \mathrm{erg} \mathrm{cm}^{-3}$ is above this threshold and about twice the pressure around ESO 137-001 (see Section 5.3.2 for a comparison of the two galaxies).

To further investigate the heated ISM hypothesis, we plot in Figure 10 the $\mathrm{H} \alpha$ surface brightness (corrected for both stellar absorption and dust extinction computed from the Balmer decrement) versus the X-ray surface brightness for the regions identified in Figure 2. Some of these regions coincide with starforming regions according to both the [O I] and [S II] MUSE diagnostic diagrams (red points), while others correspond to regions of [O I]-LINER-like emission (blue points), which are mostly classified as star-forming by $[\mathrm{S} \mathrm{II}] .{ }^{27}$ The former generally have a higher average $\mathrm{H} \alpha$ surface brightness than the latter due to the star-forming clumps.

To estimate the X-ray surface brightness of each region, we extracted the corresponding spectra by excluding the cluster contribution, then we fitted the spectra with an absorbed apec model. Due to the low statistics, we could not estimate the local properties of each region, so, under the assumption that the properties of the X-ray-emitting plasma are the same all over the galaxy, we fixed the temperature and metallicity to the values that we estimated for the whole galaxy $(k T=0.82 \mathrm{keV}$, $Z=1 Z_{\odot}$ ), and we derived the luminosity in the $0.5-8.0 \mathrm{keV}$ band from the fit normalizations that we ultimately converted in surface brightness.

Figure 10 presents some striking results. First of all, the $\mathrm{H} \alpha$ and X-ray surface brightnesses correlate as $I_{\mathrm{H}_{\alpha}} \propto I_{\mathrm{X}}^{s}$, where $I_{\mathrm{H}_{\alpha}}$ and $I_{\mathrm{X}}$ are the surface brightnesses of the two bands. This result suggests a physical relation between the two emission processes. Interestingly, the correlation is different for starforming regions and regions with an [O I] excess, with $s=0.87 \pm 0.17$ and $0.44 \pm 0.17$, respectively. In both cases, the Spearman correlation coefficients are $>0.9$. At similar $\mathrm{H} \alpha$ surface brightness, [O I]-excess regions have a higher X-ray brightness. This could be consistent with ISM heating in the [O I]-excess regions, which could account for the main observational results, explaining (a) the existence of the correlation $\mathrm{H} \alpha-\mathrm{X}$-ray and of the X-ray excess compared to star-forming regions; (b) the additional source of ionization/ excitation of the stripped gas, causing the different optical line ratios, in particular the [O I]; and (c) the lack of significant molecular gas and ongoing SF in these regions.

\footnotetext{
27 We have tried sampling smaller regions within the largest regions; the results do not change, and the relation for the diffuse component persists.
} 
Table 5

Radio Properties at $1.4 \mathrm{GHz}$

\begin{tabular}{|c|c|c|c|}
\hline Region & Flux $\left(10^{-29} \mathrm{~W} \mathrm{~Hz}^{-1} \mathrm{~m}^{-2}\right)$ & Luminosity $\left(10^{22} \mathrm{~W} \mathrm{~Hz}^{-1}\right)$ & $\overline{\mathrm{SFR}}\left(M_{\odot} \mathrm{yr}^{-1}\right)$ \\
\hline Total (without AGN) & $3.26 \pm 0.13(2.36 \pm 0.13)$ & $2.34 \pm 0.09(1.69 \pm 0.09)$ & $7.6(5.5)$ \\
\hline AGN & $0.90 \pm 0.03$ & $0.65 \pm 0.02$ & $\cdots$ \\
\hline Disk (without AGN) & $2.46 \pm 0.09(1.56 \pm 0.09)$ & $1.77 \pm 0.07(1.12 \pm 0.08)$ & $5.7(3.6)$ \\
\hline Tail & $0.8 \pm 0.16$ & $0.57 \pm 0.12$ & 1.8 \\
\hline Not compressed (without AGN) & $1.89 \pm 0.08(1.59 \pm 0.13)$ & $1.36 \pm 0.07(1.14 \pm 0.09)$ & $4.4(3.7)$ \\
\hline
\end{tabular}

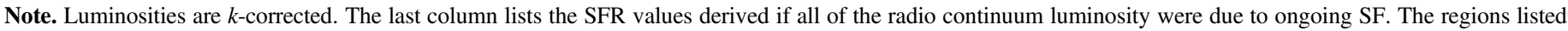

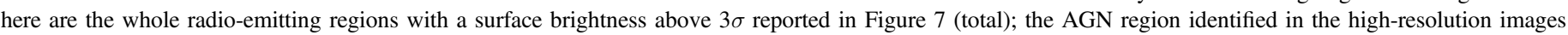

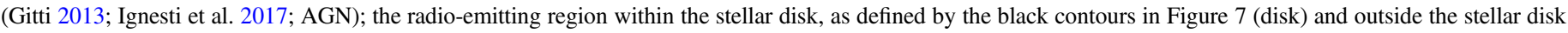
(tail); and the total when excluding the compressed region identified using the $\mathrm{H} \alpha$ contours (not compressed).

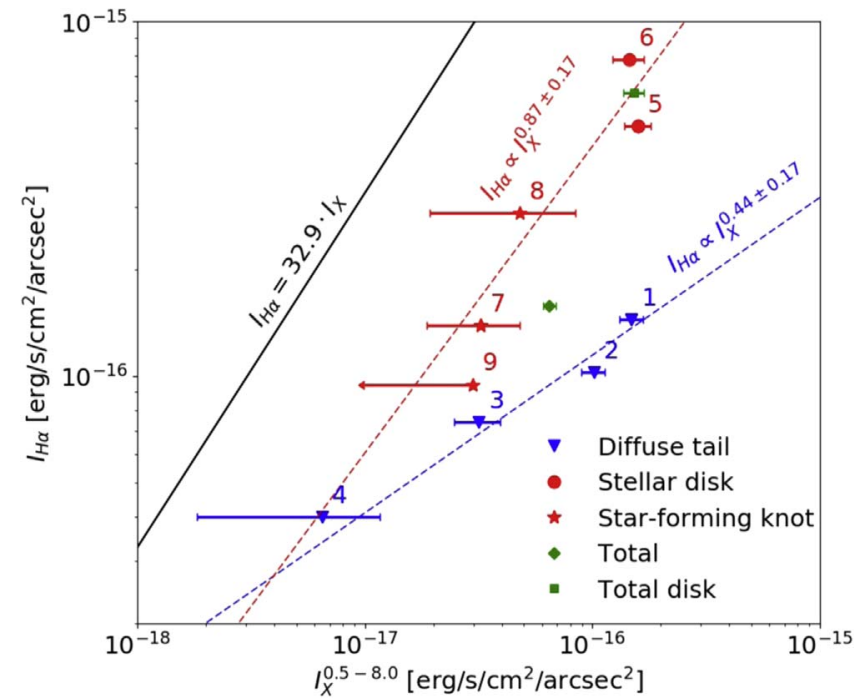

Figure 10. The $\mathrm{H} \alpha$ vs. $0.5-8 \mathrm{keV}$ X-ray surface brightness in the different regions identified with yellow polygons in Figure 2. The blue triangles are the four regions of diffuse emission with LINER-like [O I] $/ \mathrm{H} \alpha$ ratios in the right panel of Figure 2. Red indicates the regions where the $[\mathrm{O} \mathrm{I}] / \mathrm{H} \alpha$ ratio (as well as $[\mathrm{S} \mathrm{II}] / \mathrm{H} \alpha$ ) indicates SF: the star-forming regions within the disk (circles) and the knots in the tail (stars). Green shows the total and disk values. The blue dashed line is the fit to the blue diffuse emission points. The solid black line is the expected relation combining the $L_{X}=$ SFR relation from Mineo et al. (2014) and the $L_{\mathrm{H} \alpha}=$ SFR relation from Kennicutt (1998).

On the other hand, the $\mathrm{H} \alpha-\mathrm{X}$-ray surface brightness relation of even strongly star-forming regions cannot be fully explained by the expected emission at these wavelengths from a given SFR under standard conditions. In Section 5.3 we discuss how, on a global scale, there is a significant X-ray excess for the $\mathrm{H} \alpha$ emission (SFR) observed. In Figure 10, we see the same effect but on the scale of individual regions: all of the star-forming regions present an excess of X-ray emission compared to the SFR calibration commonly used in the literature, represented by the solid black line. The global values (green points in Figure 10) are dominated by the star-forming regions, especially in the disk (green square), while the overall value (green diamond) lies between the star-forming and diffuse emission regions.

At this point, we can only speculate on the possible origin for the discrepancy between the star-forming points and the standard SFR relation line in Figure 10.

(a) The standard SFR calibrations may not apply under the extraordinary physical conditions in which stars form in the tail (IMF, different timescales probed by the two indicators, etc.). To our knowledge, the $\mathrm{H} \alpha$ versus X-ray-based SFR estimates have not been tested in the literature, even for normal spirals, although given the good correlations between X-ray and radio/ $\mathrm{UV}+\mathrm{FIR}$ and between $\mathrm{H} \alpha$ and $\mathrm{UV}+\mathrm{FIR}$, a discrepancy similar to the one we observe here would be surprising in normal spirals.

(b) There could be an additional source of X-ray emission due to ISM heating, even along the line of sight of star-forming regions (though its relative importance should be lower than in the [O I]-excess regions). This is clearly possible, but the effect should be conspicuous, because the observed X-ray surface brightness of star-forming regions is a factor of 5-8 higher than expected from the $\mathrm{H} \alpha$, and in order to reconcile the two SFR estimates, the majority of the X-ray flux should arise from heating. This effect might be seen from another point of view. The main underlying relation in Figure 10 might be the one traced by the blue points, in which the emission in $\mathrm{H} \alpha$ and $\mathrm{X}$-ray arise from exactly the same process, e.g., ISM heating. The star-forming points would lie above this relation due to an excess of $\mathrm{H} \alpha$ flux due to SF.

To conclude, our data are consistent with the X-ray emission of JW100 coming from warm regions that envelope the cold ISM observed in $\mathrm{H} \alpha$ due to ISM-ICM mixing, thermal heating of the ISM due to the ICM, or shock heating. It is also consistent with the [O I] excess observed in the JW100 tail being a consequence of efficient mixing/thermal heating/ shock heating.

However, this tentative hypothesis is far from being demonstrated. Confirming or disproving it requires deeper $\mathrm{X}$-ray observations and will benefit from a comparison with the $\mathrm{H} \alpha$-X-ray surface brightness relation in other environmental conditions. For example, the diffuse LINER tail of JW100 shows interesting similarities, in terms of both optical and $\mathrm{X}$-ray properties, with the multiphase filaments observed at the centers of clusters (e.g., Werner et al. 2013). A fascinating explanation for that similarity might be that these filamentary structures, although on different scales, are generated by the motion of a substructure in the ICM (the galaxy, in our case, or an uplifting AGN cavity) that triggers the phase mixing and ICM cooling. In addition, in principle, a powerful tracer of mixing or conductive layers are UV lines, especially [O VI], which probe gas at intermediate temperatures of few $\times 10^{5} \mathrm{~K}$. This gas phase is expected to be more abundant in the heating scenario; therefore, [O VI] observations might help discriminate between the hypotheses. Moreover, cloud-scale high-resolution simulations of cold clumps embedded in a hot medium with realistic conditions for the stripped tails will be fundamental to assess the hypothesis proposed in this paper and analyze the 
various possibilities for ISM heating (e.g., Armillotta et al. 2016; Brüggen \& Scannapieco 2016; Gronke \& Oh 2018).

\subsubsection{Comparison with ESO 137-001}

We compare the X-ray properties derived in this paper with those obtained for ESO 137-001 by Sun et al. (2010). Comparisons of the SF and CO properties for JW100 and ESO 137-001 are given in Poggianti et al. (2019) and Moretti et al. (2019), respectively.

We note that the stellar mass of JW100 is almost 2 orders of magnitude higher than that of ESO 137-001. Given the low mass of ESO 137-001, this galaxy is not expected to have had a hot corona; thus, its X-ray emission must come from the interaction between cold ISM and ICM. This would suggest that the same mechanism generated the X-ray emission for JW100, giving strength to our discussion above.

The X-ray luminosity $L_{0.5-2 \mathrm{kev}}$ is $1.1 \times 10^{41} \mathrm{erg} \mathrm{s}^{-1}$ for ESO 137-001 and about twice this value for JW100 $\left((2-3.4) \times 10^{41} \mathrm{erg} \mathrm{s}^{-1}\right.$; Table 4). The morphologies of the X-ray tails of the two galaxies are very different: that of JW100, at the current depth of the Chandra data, is within the galaxy tidal truncation radius (truncated by the cluster potential, about $38 \mathrm{kpc}$ ), while the observed $80 \mathrm{kpc}$ X-ray tail of ESO 137-001 is well outside of its truncation radius. However, we calculate that with the exposure time and the Chandra effective area of the Sun et al. observations (given the degrading of Chandra performance with time), we would have had 2.5 times the counts we observed. Given the $\mathrm{H} \alpha-\mathrm{X}$-ray correlation we observe, it is reasonable to expect the X-ray tail to be as extended as the $\mathrm{H} \alpha$ tail. Deeper X-ray observations would be needed to observe the full extent of the X-ray tail in JW100.

Interestingly, the X-ray temperature derived for the two galaxies with apec+apec models is similar ( 0.7 versus $0.8 \mathrm{keV}$; to be compared with the typical temperature of hot gas in normal spirals of $\sim 0.3 \mathrm{keV}$; Strickland et al. 2004; Mineo et al. 2012b). Since the temperatures of the two galaxies are almost the same, it is tempting to conclude that the physical mechanism that generates the X-ray emission is the same: heating of cold ISM. The ICM ambient temperature is higher in ESO 137-001 (6 keV) than in JW100 (3.5 keV), while both the gas density and the thermal pressure are higher for JW100: $3.2 \times 10^{-3}$ versus $(1-1.4) \times 10^{-3} \mathrm{~cm}^{-3}$ and $3 \times 10^{-11}$ versus $1.8 \times 10^{-11} \mathrm{erg} \mathrm{cm}^{-3}$. The ram pressure at the galaxy location

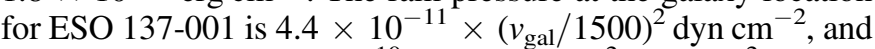
for JW100, it is $1.3 \times 10^{-10} \times\left(v_{\mathrm{gal}} / 1500\right)^{2} \mathrm{dyn} \mathrm{cm}^{-2}$; thus, it is $\geqslant 1.9 \times 10^{-10} \mathrm{dyn} \mathrm{cm}^{-2}$ (see the Appendix), given that the galaxy peculiar velocity observed with MUSE $\left(1807 \mathrm{~km} \mathrm{~s}^{-1}\right)$ significantly underestimates its total speed.

\section{X-Ray Point Sources: AGN and ULX Candidate}

\section{1. $A G N$}

We extract the X-ray AGN spectrum using a background that includes the cluster contribution at the position of the galaxy. ${ }^{28}$ We compute the hardness ratio (HR), defined as the ratio $\frac{\mathrm{H}-\mathrm{S}}{\mathrm{H}+\mathrm{S}}$, where $\mathrm{H}$ and $\mathrm{S}$ are the net counts of the source in the hard $(2-7 \mathrm{keV})$ and soft $(0.5-2 \mathrm{keV})$ bands. We find

\footnotetext{
${ }^{28}$ From the inset in Figure 4, we see that, even using a small region for the extraction of the counts, we expect a residual contamination from the galaxy emission itself, which, however, is not significant in the counts.
}

$\mathrm{HR}=-0.3 /-0.10$ in the two observations, respectively, to be compared with the mean ratio for type 2 AGNs, $\mathrm{HR}=-0.03 \pm 0.46$ from Marchesi et al. (2016). This source is therefore fully consistent with being a low-luminosity, absorbed AGN, as also confirmed by the shape of the spectrum, which shows two peaks. We fit the spectrum with a simple combination of a power-law model plus an absorbed power-law model, fixing the two slopes to be the same, with absorption fixed to the Galactic value of $3.8 \times 10^{20} \mathrm{~cm}^{-2}$ (Kalberla et al. 2005; phabs* (pow+phabs+pow) in xspec). This shape is a simple representation of a direct plus a reflected component, as in an obscured AGN. The resulting slope is $\Gamma=2.5$ with a very large uncertainty. ${ }^{29}$ The spectral shape, with a slope steeper than the average slope for Seyfert $2 \mathrm{~s}$, is nevertheless very similar to that of the brighter $\left(L_{X} \sim 10^{43} \mathrm{erg} \mathrm{s}^{-1}\right)$ Seyfert 2 AXJ $2254+1146$ (Della Ceca et al. 2000), although the statistics here is not enough to detect a possible iron line, as expected in a Seyfert 2 . The absorbing column is of the order of $3 \times 10^{23} \mathrm{~cm}^{-2}$, and the direct component is about $1 \%$ of the reflected one. The total X-ray luminosity of the AGN is $L_{X}$ $(0.5-10)=2.4 \times 10^{41} \mathrm{erg} \mathrm{s}^{-1}$.

The intensity variability in the $10 \mathrm{yr}$ between the two observations is smaller than $10 \%$, comparable with the statistical uncertainty on the count rate.

\subsection{ULX Candidate}

The second, southern X-ray point source (see Table 2) is detected in the X-ray tail, just outside the stellar body of the galaxy. Fitting the spectrum with a power law (phabs*pow in xspec), again using the Galactic absorption value of $3.8 \times 10^{20} \mathrm{~cm}^{-2}$ (Kalberla et al. 2005), we get a slope $\Gamma=$ $1.69[1.44-1.96]$ with a $\chi_{\nu}^{2} /$ dof $=1.4 / 8$. The corresponding unabsorbed flux $(0.5-2 \mathrm{keV})$ is $3.6 \times 10^{-15} \mathrm{erg} \mathrm{s}^{-1} \mathrm{~cm}^{-2}$. The flux in the two observations is consistent with no variability.

The inset in Figure 4 shows the three X-ray color images of the galaxy. The two point sources are much harder than the diffuse emission. The AGN is embedded in the galactic emission, while the southern source appears at the edge of the diffuse emission.

We first investigate the possibility that the source is not related to the galaxy. The total number of expected contaminants from the resolved X-ray background $\log \mathrm{N}-\log \mathrm{S}$ (Moretti et al. 2003) at the flux of the source is only 0.027 in an area of $40^{\prime \prime} \times 30^{\prime \prime}$, corresponding to the region occupied by the $\mathrm{H} \alpha$ emission of the galaxy. It is therefore unlikely that this is a background source. Moreover, there is no optical counterpart visible in the MUSE white-light image, i.e., collapsing all of the MUSE wavelength range, down to a magnitude of $V \leqslant 24$. Therefore, we can exclude the bulk of the AGN counterparts that should have visual magnitudes between 22 and 24 (Maccacaro et al. 1998). Furthermore, at the spatial location of this X-ray source, there is no emission line detected in the MUSE data (above the noise) that would reveal the presence of an X-ray-bright background/foreground source.

We also exclude foreground Galactic stars as interlopers; normal, X-ray-emitting stars should be brighter than 20 mag in the optical and would be seen by MUSE, while accreting neutron stars (that would have an X-ray over $V$-band flux ratio $f_{X} / f_{V} \geqslant 1000$, corresponding to $\left.m_{V}=30\right)$ are very rare, especially at high galactic latitude $\left(b^{\mathrm{II}}=-38^{\circ}\right)$.

\footnotetext{
${ }^{29}$ The results do not change significantly by fixing the slope to $\Gamma=1.7$.
} 
We are left, then, with the interesting possibility that the source is associated with JW100 itself. At the luminosity distance of the cluster, the detected flux corresponds to $L_{X}(0.3-10)=7.8 \times 10^{40} \mathrm{erg} \mathrm{s}^{-1}$. Such a high X-ray luminosity in a pointlike source located close to star-forming regions makes the source a ULX, which is expected to be produced by recent strong SF episodes (see, e.g., Kaaret et al. 2017 for a review of ULXs). The ULX would have formed during the SF enhancement induced by ram pressure. Interestingly, the ULX candidate is at the location of a bright UV knot (see Figure 7) and close to an $\mathrm{H} \alpha$ clump. Thus, our data show a spatial association between the ULX candidate and a recently formed bright stellar clump. At a distance $D=255 \mathrm{Mpc}$, this is the farthest ULX candidate found and a very luminous one, although not enough to enter the class of hyperluminous X-ray sources, i.e., the ULXs with luminosity in excess of $10^{41} \mathrm{erg} \mathrm{s}^{-1}$ (see, e.g., Gao et al. 2003; Wolter \& Trinchieri 2004 for Cartwheel N10 and Farrell et al. 2009 for HLX-1 in ESO 24349). The X-ray spectrum is consistent with what is seen in other ULXs with this level of statistics (e.g., Wolter \& Trinchieri 2004; Swartz et al. 2011).

The bulk of ULXs are consistent with being HMXBs. We can use the relations from Mineo et al. (2012a) concerning the number of HMXB sources and the total X-ray luminosity expected as a function of SFR. Using SFR $=4.0 M_{\odot} \mathrm{yr}^{-1}$ (see Section 2), the total number of expected bright sources is $N_{\text {HMXB }}\left(>10^{39}\right)=0.49 \times \mathrm{SFR} \sim 2$, which is consistent with our observation of a bright ULX. However, the expected total luminosity in $\mathrm{HMXBs,} \mathrm{of} \mathrm{which} \mathrm{the} \mathrm{majority} \mathrm{are} \mathrm{unresolved}$ in this observation, is $L_{\mathrm{X}}^{\mathrm{HMXB}}=2.6 \times 10^{39} \times \mathrm{SFR} \mathrm{erg} \mathrm{s}^{-1}=$ $1.0 \times 10^{40} \mathrm{erg} \mathrm{s}^{-1}$. The detected ULX is already almost an order of magnitude more luminous than the total $L_{X}$ luminosity expected outside the scatter in the relation $(\sigma=0.43 \mathrm{dex})$.

In the galaxy ESO 137-001, Sun et al. (2010) identified six ULXs with $L_{0.3-10 \mathrm{keV}}$ up to $2.5 \times 10^{40} \mathrm{erg} \mathrm{s}^{-1}$ and thus much fainter than our candidate ULX in JW100. The SFR of ESO $137-001$ is also at least an order of magnitude smaller than that in JW100, and interestingly, the total X-ray luminosity in the point sources is larger than expected in both galaxies.

The $N$-body/smoothed particle hydrodynamics simulations run with GADGET-2 have shown that both ram pressure and viscous transfer effects are necessary to produce the large number of ULXs seen in the interacting galaxy NGC 2276, which falls in the potential well and ICM of the NGC 2300 group (Wolter et al. 2015). We could be witnessing a similar effect here, with ram pressure enhancing the efficiency of the SF process and the luminosity of the resulting binary system. A similar effect was also found for another extreme environment, that of collisional ring galaxies, in which both the total number of ULXs and the number of ULXs per unit SFR are observed to be in the upper envelope of the normal galaxy distribution (Wolter et al. 2018). All of the evidence suggests a flattening of the X-ray luminosity function when local SF enhancements/ bursts occur due to gravitational interactions and/or stripping, although statistically strong conclusions cannot be drawn due to the relatively small number of ULXs observed.

Finally, if we are indeed witnessing an HMXB, we can confirm that the onset of the interaction with the ICM that triggered the SF episode happened not more than a few hundred Myr ago, given the lifetime of the donor star involved in an HMXB.

\section{Summary}

In this paper, we have studied the jellyfish galaxy JW100, which presents a striking extraplanar tail of multiphase gas due to ram pressure stripping from the ICM of the A2626 cluster. This work is part of an ongoing effort to understand the physical processes that create tails observable at different wavelengths, as well as the baryonic cycle in the tails and disks of jellyfish galaxies.

We use the multiwavelength data set of the GASP survey, which consists of optical integral field spectroscopy from MUSE, X-ray ACIS-S Chandra data, $1.4 \mathrm{GHz}$ observations from the VLA, NUV imaging from UVIT on board AstroSat, and CO(2-1) ALMA observations. These data offer a detailed and comprehensive view of the ionized gas, X-ray gas, molecular gas, stellar UV light, and radio continuum light emitted from the tail and disk. The spatial resolution of these observations samples an $\sim 1 \mathrm{kpc}$ scale, except for the radio continuum observations that have a resolution of $\sim 4 \times 3.5 \mathrm{kpc}$.

Our main results can be summarized as follows.

1. The ICM at the clustercentric distance of JW100 has $k T=3.5 \mathrm{keV}$, a metallicity 0.35 solar, and a density $\rho_{\mathrm{ICM}}=5.8 \times 10^{-27} \mathrm{~g} \mathrm{~cm}^{-3}$. The X-ray emission of the JW100 region can be equally well modeled by adding to the ICM component either an absorbed, thermal, singletemperature component $(k T=0.82 \mathrm{keV})$ or a multiphase, multitemperature model. The galaxy is moving supersonically (Mach number $\sim 2$ ), but the presence of a bow shock as inferred by an X-ray temperature break remains unconfirmed until more sensitive observations are obtained.

2. The $50 \mathrm{kpc}$ long $\mathrm{H} \alpha$ tail presents bright clumps embedded in diffuse emission. The former are giant and supergiant star-forming regions, mostly located in the southern part of the tail. All of the MUSE optical line ratios in these clumps indicate that the gas is photoionized by young massive stars. The ionization source of most of the diffuse emission, instead, is SF, according to the $[\mathrm{S}$ II] diagnostic diagram, but presents an excess of [O I]-LINER-like emission.

3. Molecular gas is present in the disk (where $\mathrm{H} \alpha$ is also observed) and large complexes in the tail, mostly in the southern part of the tail. A detailed analysis of the ALMA CO emission is given in Moretti et al. (2019).

4. The $\mathrm{H} \alpha$ clumps, CO clumps, and NUV emission are all linked to in situ SF in the tail that currently mostly takes place in the southern part of the tail. On small scales (1 to a few kpc), we observe regions with $\mathrm{H} \alpha, \mathrm{CO}$, and UV but also regions with only bright $\mathrm{CO}$ emission, only $\mathrm{H} \alpha$ and $\mathrm{UV}$, or only UV. We interpret this as an SF sequence, in which SF progresses from the molecular cloud phase with no stars formed yet, to later stages where the molecular gas has already been dispersed by the stars formed, until only the UV light of young stars is still visible. This evolutionary sequence corresponds to a spatial sequence in the stripped tail, going from further away to closer to the disk.

5. The radio continuum emission of JW100 is mostly nonthermal and it is synchrotron emission of relativistic electrons. This indicates the presence of magnetic fields in the stripped tails (see A. Mueller et al. 2019, in preparation, for a direct measurement of the magnetic field in a jellyfish 
tail) and is consistent with supernovae forming in the stripped tails. The observed $1.4 \mathrm{GHz}$ flux is consistent with that expected from the SFR measured from $\mathrm{H} \alpha$ for standard IMF assumptions, though a contribution from other sources, such as stripping of relativistic electrons, cannot be excluded. The spatial distribution of the radio emission, however, does not coincide with the currently star-forming regions: the former is mostly in the northern part of the tail, and in the southern part, the radio avoids the $\mathrm{H} \alpha / \mathrm{CO}$ clumps. This could be due to the lifetime of the electrons (longer than the $\mathrm{H} \alpha$ timescale), during which they can travel large distances, and/or to supernova explosions not having occurred yet in the youngest star-forming regions.

6. In contrast with the SF tracers, the extraplanar X-ray emission is mostly in the northern part of the tail. We find that this X-ray emission cannot be explained by SF (HMXBs and ISM ionized by supernovae and massive stars), because the SFR derived in this case would be between 4 and 30 times higher than the SFR derived from $\mathrm{H} \alpha$. The X-ray luminosity observed is similar to the one expected for the hot X-ray halo of a galaxy as massive as JW100, but a simple model rules out the stripped hot halo as the origin of the X-ray tail based on timescale arguments. We conclude that a significant fraction of the X-ray emission of JW100 must arise from heating of its stripped ISM, due to mixing of the ISM and the ICM, thermal conduction from the ICM, or shock heating.

7. We find a striking double correlation between the $\mathrm{H} \alpha$ and $\mathrm{X}$-ray surface brightness. The correlation is shallower in regions of diffuse, [O I]-LINER-like emission $\left(I_{\mathrm{H} \alpha} \propto I_{X}^{0.44}\right)$ and steeper in star-forming regions of the disk and tail $\left(I_{\mathrm{H} \alpha} \propto I_{X}^{0.87}\right)$. Even in star-forming regions, the X-ray brightness significantly exceeds the one expected from $\mathrm{H} \alpha$ assuming the standard calibrations between SFR and $\mathrm{H} \alpha$ / UV.

This result corroborates the scenario in which the stripped ISM is heated due to the interaction with the ICM (either mixing, thermal conduction, or shocks). This heating could be responsible for (a) much of the X-ray emission, (b) the [O I] excess observed in the diffuse gas of the tail, or (c) the lack of SF where such heating is more efficient (i.e., the northern part of the tail). Where the heating is less efficient (in the southern part of the tail, with little or no X-ray emission), SF occurs in giant clumps with molecular gas.

8. The southern point source is most likely a very bright, and thus rare, ULX, with a luminosity $\left(L_{X}(0.3-10)=7.8 \times\right.$ $10^{40} \mathrm{erg} \mathrm{s}^{-1}$ ) that places it at the bright end of the ULX luminosity function. Since ram pressure in a dense fluid (as well as gravitational interactions) can enhance the production of bright X-ray sources, we deem this a valid explanation for the data in hand. As an HMXB (in which the donor star has a short lifetime), it would have formed during the SF episode triggered at that location by the ram pressure exerted by the ICM a few hundred Myr before the observations, consistent with all other evidence for ram pressure stripping in JW100.

Multiwavelength studies of jellyfish galaxies, such as the one we have presented here for JW100 and those in the literature for ESO 137-001, are powerful probes for a variety of physical processes, including SF under extreme environmental conditions and the interplay between the ISM and intergalactic medium. Hence, jellyfish galaxies can be a laboratory of physics for circumgalactic medium (thus galaxy evolution) studies in general. Fundamental open questions remain unanswered by our results, including how gas can cool and form new stars in the ICMembedded tails and the physical mechanism of heating of the stripped ISM (how important is mixing versus thermal conduction versus shocks). Multiwavelength studies for a larger number of jellyfish galaxies for different local ICM conditions, coupled with hydrodynamical simulations, offer the prospect of significant advancement in these fields.

We thank Emiliano Munari for providing us with the CLUMPS code in advance of publication and Luca Zampieri and Fabrizio Nicastro for useful discussions regarding the ULX. We are grateful to the anonymous referee who helped us strengthen and clarify the paper. Based on observations collected at the European Organization for Astronomical Research in the Southern Hemisphere under ESO program 196.B-0578. This paper makes use of the following ALMA data: ADS/JAO. ALMA\#2017.1.00496.S. ALMA is a partnership of the ESO (representing its member states), NSF (USA), and NINS (Japan), together with the NRC (Canada) and NSC and ASIAA (Taiwan), in cooperation with the Republic of Chile. The Joint ALMA Observatory is operated by the ESO, AUI/NRAO, and NAOJ. This publication uses the data from the AstroSat mission of the Indian Space Research Organisation (ISRO), archived at the Indian Space Science Data Centre (ISSDC). This project has received funding from the European Research Council (ERC) under the European Union's Horizon 2020 research and innovation program (grant agreement No. 833824). We acknowledge financial support from PRIN-SKA 2017 (PI: L. Hunt). We acknowledge financial contributions from the agreement ASIINAF No. 2017-14-H.0 (PI: Moretti) and the INAF mainstream funding program (PI: Vulcani). E.R. acknowledges the support of the STFC through the University of Hull's consolidated grant ST/R000840/1. A.W. acknowledges financial contributions from the agreement ASI-INAF No. 2017-14-H.0. B.V. and M.G. acknowledge the Italian PRIN-MIUR 2017 (PI: Cimatti). This work made use of the KUBEVIZ software, which is publicly available at http://www.mpe.mpg.de/ dwilman/kubeviz/.

\section{Appendix \\ A Model for the Stripping of the Hot Halo}

We can test whether the observed X-ray tail can be due to a hot stripped halo with a simple model. According to McCarthy et al. (2008), the ICM ram pressure strips the halo gas (assumed to be spherically distributed) at a projected radius $R$ if

$$
P_{\text {ram }}=\rho_{\mathrm{ICM}} v_{\mathrm{ICM}}^{2}>g_{\max }(R) \sigma_{\text {halo }}(R),
$$

where $\rho_{\text {ICM }}=5.8 \times 10^{-27} \mathrm{~g} \mathrm{~cm}^{-3}$ is the local ICM density and $v_{\mathrm{ICM}}$ is the relative velocity between JW100 and the ICM, here assumed to lie in the range between 1800 (the observed line-of-sight velocity) and $2500 \mathrm{~km} \mathrm{~s}^{-1}$ (assumed as fiducial maximum velocity). The quantities on the right-hand side of the equation are the maximum gravitational acceleration component parallel to $v_{\mathrm{ICM}}$ at the projected radius $R$, and $\sigma_{\text {halo }}(R)$ is the surface density $\left(\mathrm{g} \mathrm{cm}^{-2}\right)$ of the hot halo gas.

In order to estimate $g_{\max }(R)$ and the distribution of the halo gas, we need to build a mass model for the galaxy. Our fiducial model includes two stellar components (a stellar bulge and a stellar disk) and a dark matter halo. The bulge, approximated 


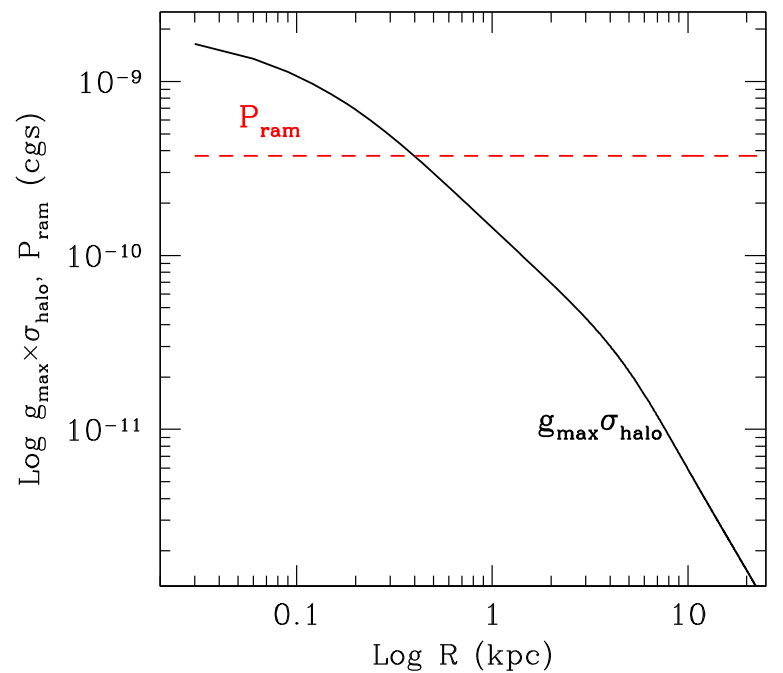

Figure 11. The ICM ram pressure (red dashed line) versus the component along the direction of motion of the restoring force per unit area (solid black line) for the fiducial model with the Miyamoto-Nagai disk.

with a Hernquist profile (Hernquist 1990), has mass $M_{b}=$ $3 \times 10^{10} M_{\odot}$ and half-mass radius $r_{1 / 2}=1.2 \mathrm{kpc}$. The disk has a very flattened (Miyamoto \& Nagai 1975) distribution with mass $M_{d}=2.5 \times 10^{11} M_{\odot}$ and scale parameters $a=6$ and $b=0.5 \mathrm{kpc}$ (see also Binney \& Tremaine 1987). The total stellar mass is thus $M_{*}=2.8 \times 10^{11} M_{\odot}$.

Finally, the dark halo assumes a Navarro et al. (1996) shape, with total mass $M_{\mathrm{DM}}=1.2 \times 10^{13} M_{\odot}$ and a concentration $c=9.8$.

The hot gas is initially set in hydrostatic equilibrium in the total potential, assuming a constant temperature $T_{\text {halo }}=5.8 \times$ $10^{6} \mathrm{~K}\left(k T_{\text {halo }}=0.5 \mathrm{keV}\right)$, chosen to agree with the mean halo temperature of NGC 1961 (Anderson et al. 2016). The central halo gas density is also chosen for the gas density to agree with the NGC 1961 profile (Anderson et al. 2016). The 3D halo density profile is then integrated to get the surface density $\sigma_{\text {halo }}(R)$.

With this simple model, we can use the equation above to calculate the projected radius $R$ beyond which the stripping is effective. Figure 11 shows that for $R \geqslant 380 \mathrm{pc}$, the ram pressure is larger than the restoring force per square centimeter. The timescale to significantly alter the distribution of the halo gas is $\tau_{\text {strip }}=R / v_{\text {ICM }} \sim 0.5(R / \mathrm{kpc})\left(v / 2000 \mathrm{~km} \mathrm{~s}^{-1}\right)^{-1}$ Myr. Therefore, the halo gas is advected by $\sim 30 \mathrm{kpc}$ (the size of the tail) in $\sim 15$ Myr. From these numbers, we expect that the ram pressure quickly disrupted and removed most or all of the hot halo, well before the galaxy reached the current location, even taking into account the lower ram pressure experienced by JW100 in the past while traveling through lower-density ICM regions.

In order to gauge the uncertainty in the mass model, we also considered a similar model as above, replacing the MiyamotoNagai disk with a thin Kuzmin disk of total mass $M_{d}^{\mathrm{Kuz}}=$ $2 \times 10^{11} M_{\odot}$ and scale parameter $a=2.5 \mathrm{kpc}$ (see Binney \& Tremaine 1987). In this case, the ram pressure is able to quickly strip the halo gas beyond $R \sim 950$ pc.

In addition, on top of the classical ram pressure stripping investigated above, viscous or turbulent ablation (Nulsen 1986) would help the gas removal process. To conclude, we expect the hot galaxy halo to be removed on very short timescales, making the hot halo origin of the observed X-ray tail unplausible.

\section{ORCID iDs}

Bianca M. Poggianti (D) https://orcid.org/0000-00018751-8360

Myriam Gitti (i) https://orcid.org/0000-0002-0843-3009

Andrea Biviano (iD https://orcid.org/0000-0002-0857-0732

Koshy George (iD https://orcid.org/0000-0002-1734-8455

Benedetta Vulcani (i) https://orcid.org/0000-0003-0980-1499

Marco Gullieuszik (1) https://orcid.org/0000-0002-7296-9780

Alessia Moretti (i) https://orcid.org/0000-0002-1688-482X

Daniela Bettoni (iD https://orcid.org/0000-0002-4158-6496

Yara L. Jaffé (iD https://orcid.org/0000-0003-2150-1130

Neven Tomičić (iD https://orcid.org/0000-0002-8238-9210

Stephanie Tonnesen (iD https://orcid.org/0000-0002-

8710-9206

Callum Bellhouse (iD https://orcid.org/0000-0002-6179-8007

Jacopo Fritz (iD https://orcid.org/0000-0002-7042-1965

\section{References}

Agrawal, P. C. 2006, AdSpR, 38, 2989

Anderson, M. E., Churazov, E., \& Bregman, J. N. 2016, MNRAS, 455, 227

Armillotta, L., Fraternali, F., \& Marinacci, F. 2016, MNRAS, 462, 4157

Arnaud, K. A. 1996, in ASP Conf. Ser. 101, Astronomical Data Analysis Software and Systems V, ed. G. H. Jacoby \& J. Barnes (San Francisco, CA: ASP), 17 Bacon, R., Accardo, M., Adjali, L., et al. 2010, Proc. SPIE, 7735, 773508

Bekki, K. 2009, MNRAS, 399, 2221

Bell, E. F. 2003, ApJ, 586, 794

Bell, E. F., \& de Jong, R. S. 2001, ApJ, 550, 212

Bellhouse, C., Jaffé, Y. L., McGee, S. L., et al. 2019, MNRAS, 485, 1157

Binney, J., \& Tremaine, S. 1987, Galactic Dynamics (Princeton, NJ: Princeton Univ. Press)

Biviano, A., Moretti, A., Paccagnella, A., et al. 2017, A\&A, 607, A81

Biviano, A., Rosati, P., Balestra, I., et al. 2013, A\&A, 558, A1

Boselli, A., Cuillandre, J. C., Fossati, M., et al. 2016, A\&A, 587, A68

Boselli, A., \& Gavazzi, G. 2006, PASP, 118, 517

Bregman, J. N., Anderson, M. E., Miller, M. J., et al. 2018, ApJ, 862, 3

Brüggen, M., \& Scannapieco, E. 2016, ApJ, 822, 31

Chabrier, G. 2003, PASP, 115, 763

Chung, A., van Gorkom, J. H., Kenney, J. D. P., \& Vollmer, B. 2007, ApJL, 659, L115

Consolandi, G., Gavazzi, G., Fossati, M., et al. 2017, A\&A, 606, A83

Della Ceca, R., Maccacaro, T., Rosati, P., \& Braito, V. 2000, A\&A, 355, 121

Ebeling, H., Ma, C.-J., \& Barrett, E. 2014, ApJS, 211, 21

Erwin, P., Pohlen, M., \& Beckman, J. E. 2008, AJ, 135, 20

Farrell, S. A., Webb, N. A., Barret, D., Godet, O., \& Rodrigues, J. M. 2009, Natur, 460, 73

Fossati, M., Fumagalli, M., Boselli, A., et al. 2016, MNRAS, 455, 2028

Fossati, M., Fumagalli, M., Gavazzi, G., et al. 2019, MNRAS, 484, 2212

Freeman, K. C. 1970, ApJ, 160, 811

Fritz, J., Moretti, A., Gullieuszik, M., et al. 2017, ApJ, 848, 132

Fumagalli, M., Fossati, M., Hau, G. K. T., et al. 2014, MNRAS, 445, 4335

Fumagalli, M., Gavazzi, G., Scaramella, R., \& Franzetti, P. 2011, A\&A, 528, A46

Gao, Y., Wang, Q. D., Appleton, P. N., \& Lucas, R. A. 2003, ApJL, 596, L171 Girish, V., Tandon, S. N., Sriram, S., Kumar, A., \& Postma, J. 2017, ExA, 43,59

Gitti, M. 2013, MNRAS, 436, L84

Gitti, M., Brunetti, G., Feretti, L., \& Setti, G. 2004, A\&A, 417, 1

Gronke, M., \& Oh, S. P. 2018, MNRAS, 480, L111

Gullieuszik, M., Poggianti, B. M., Moretti, A., et al. 2017, ApJ, 846, 27

Hernquist, L. 1990, ApJ, 356, 359

Hester, J. A., Seibert, M., Neill, J. D., et al. 2010, ApJL, 716, L14

Ignesti, A., Gitti, M., Brunetti, G., et al. 2018, A\&A, 610, A89

Ignesti, A., Gitti, M., Brunetti, G., Feretti, L., \& Giovannini, G. 2017, A\&A, 604, A21

Jachym, P., Kenney, J. D. P., Sun, M., et al. 2019, ApJ, 883, 145

Jáchym, P., Sun, M., Kenney, J. D. P., et al. 2017, ApJ, 839, 114

Jaffé, Y. L., Poggianti, B. M., Moretti, A., et al. 2018, MNRAS, 476, 4753 
Kaaret, P., Feng, H., \& Roberts, T. P. 2017, ARA\&A, 55, 303

Kadam, S. K., Sonkamble, S. S., Pawar, P. K., \& Patil, M. K. 2019, MNRAS, 484, 4113

Kalberla, P. M. W., Burton, W. B., Hartmann, D., et al. 2005, A\&A, 440, 775

Kale, R., \& Gitti, M. 2017, MNRAS, 466, L19

Kenney, J. D. P., Geha, M., Jáchym, P., et al. 2014, ApJ, 780, 119

Kenney, J. D. P., van Gorkom, J. H., \& Vollmer, B. 2004, AJ, 127, 3361

Kennicutt, R. C., Jr. 1998, ARA\&A, 36, 189

Kruijssen, J. M. D., Schruba, A., Chevance, M., et al. 2019, Natur, 569, 519

Lang, D., Hogg, D. W., Mierle, K., Blanton, M., \& Roweis, S. 2010, AJ, 139,1782

Lee, B., Chung, A., Tonnesen, S., et al. 2017, MNRAS, 466, 1382

Li, J.-T., Bregman, J. N., Wang, Q. D., Crain, R. A., \& Anderson, M. E. 2016, ApJ, 830, 134

Maccacaro, T., Caccianiga, A., della Ceca, A., Wolter, A., \& Gioia, I. M. 1998, AN, 319, 15

Marchesi, S., Civano, F., Elvis, M., et al. 2016, ApJ, 817, 34

McCarthy, I. G., Frenk, C. S., Font, A. S., et al. 2008, MNRAS, 383, 593

McKee, C. F., \& Begelman, M. C. 1990, ApJ, 358, 392

Merluzzi, P., Busarello, G., Dopita, M. A., et al. 2013, MNRAS, 429, 1747

Mineo, S., Gilfanov, M., Lehmer, B. D., Morrison, G. E., \& Sunyaev, R. 2014, MNRAS, 437, 1698

Mineo, S., Gilfanov, M., \& Sunyaev, R. 2012a, MNRAS, 419, 2095

Mineo, S., Gilfanov, M., \& Sunyaev, R. 2012b, MNRAS, 426, 1870

Miyamoto, M., \& Nagai, R. 1975, PASJ, 27, 533

Moretti, A., Campana, S., Lazzati, D., \& Tagliaferri, G. 2003, ApJ, 588, 696

Moretti, A., Gullieuszik, M., Poggianti, B., et al. 2017, A\&A, 599, A81

Moretti, A., Paladino, R., Poggianti, B. M., et al. 2019, ApJ, submitted

Moretti, A., Poggianti, B. M., Fasano, G., et al. 2014, A\&A, 564, A138

Moretti, A., Poggianti, B. M., Gullieuszik, M., et al. 2018, MNRAS, 475, 4055

Murphy, E. J., Kenney, J. D. P., Helou, G., Chung, A., \& Howell, J. H. 2009, ApJ, 694, 1435

Navarro, J. F., Frenk, C. S., \& White, S. D. M. 1996, ApJ, 462, 563

Nulsen, P. E. J. 1986, MNRAS, 221, 377

Poggianti, B. M., Fasano, G., Omizzolo, A., et al. 2016, AJ, 151, 78

Poggianti, B. M., Gullieuszik, M., Tonnesen, S., et al. 2019, MNRAS, 482, 4466

Poggianti, B. M., Jaffé, Y. L., Moretti, A., et al. 2017a, Natur, 548, 304

Poggianti, B. M., Moretti, A., Gullieuszik, M., et al. 2017b, ApJ, 844, 48
Poggianti, B. M., Smail, I., Dressler, A., et al. 1999, ApJ, 518, 576

Postma, J. E., \& Leahy, D. 2017, PASP, 129, 115002

Radovich, M., Poggianti, B., Jaffé, Y. L., et al. 2019, MNRAS, 486, 486

Ranalli, P., Comastri, A., \& Setti, G. 2003, A\&A, 399, 39

Rasmussen, J., Ponman, T. J., \& Mulchaey, J. S. 2006, MNRAS, 370, 453

Rich, J. A., Kewley, L. J., \& Dopita, M. A. 2011, ApJ, 734, 87

Roediger, E., Brüggen, M., Owers, M. S., Ebeling, H., \& Sun, M. 2014, MNRAS, 443, L114

Schellenberger, G., \& Reiprich, T. H. 2015, A\&A, 583, L2

Singh, K. P., White, N. E., \& Drake, S. A. 1996, ApJ, 456, 766

Sivanandam, S., Rieke, M. J., \& Rieke, G. H. 2010, ApJ, 717, 147

Sivanandam, S., Rieke, M. J., \& Rieke, G. H. 2014, ApJ, 796, 89

Smith, R. J., Lucey, J. R., Hammer, D., et al. 2010, MNRAS, 408, 1417

Strickland, D. K., Heckman, T. M., Colbert, E. J. M., Hoopes, C. G., \& Weaver, K. A. 2004, ApJS, 151, 193

Sun, M., Donahue, M., Roediger, E., et al. 2010, ApJ, 708, 946

Sun, M., Donahue, M., \& Voit, G. M. 2007, ApJ, 671, 190

Sun, M., \& Vikhlinin, A. 2005, ApJ, 621, 718

Swartz, D. A., Soria, R., Tennant, A. F., \& Yukita, M. 2011, ApJ, 741, 49

Tabatabaei, F. S., Schinnerer, E., Krause, M., et al. 2017, ApJ, 836, 185

Tandon, S. N., Hutchings, J. B., Ghosh, S. K., et al. 2017a, JApA, 38, 28

Tandon, S. N., Subramaniam, A., Girish, V., et al. 2017b, AJ, 154, 128

Tiret, O., Combes, F., Angus, G. W., Famaey, B., \& Zhao, H. S. 2007, A\&A, 476, L1

Tonnesen, S., Bryan, G. L., \& Chen, R. 2011, ApJ, 731, 98

Vollmer, B., Huchtmeier, W., \& van Driel, W. 2005, A\&A, 439, 921

Vulcani, B., Poggianti, B. M., Moretti, A., et al. 2018, ApJ, 852, 94

Werner, N., McNamara, B. R., Churazov, E., \& Scannapieco, E. 2019, SSRv, 215,5

Werner, N., Oonk, J. B. R., Canning, R. E. A., et al. 2013, ApJ, 767, 153

Wolter, A., Esposito, P., Mapelli, M., Pizzolato, F., \& Ripamonti, E. 2015, MNRAS, 448, 781

Wolter, A., Fruscione, A., \& Mapelli, M. 2018, ApJ, 863, 43

Wolter, A., \& Trinchieri, G. 2004, A\&A, 426, 787

Wong, K.-W., Sarazin, C. L., Blanton, E. L., \& Reiprich, T. H. 2008, ApJ, 682,155

Wong, O. I., Kenney, J. D. P., Murphy, E. J., \& Helou, G. 2014, ApJ, 783, 109

Yagi, M., Yoshida, M., Komiyama, Y., et al. 2010, AJ, 140, 1814

Yoshida, M., Yagi, M., Komiyama, Y., et al. 2008, ApJ, 688, 918 